\title{
The $\kappa-\mu$ / Inverse Gamma and $\eta-\mu$ / Inverse Gamma Composite Fading Models: Fundamental Statistics and Empirical Validation
}

\author{
Seong Ki Yoo, Member, IEEE, Nidhi Bhargav, Student Member, IEEE, \\ Simon L. Cotton, Senior Member, IEEE, Paschalis C. Sofotasios, Senior Member, IEEE, \\ Michail Matthaiou, Senior Member, IEEE, Mikko Valkama, Senior Member, IEEE, \\ and George K. Karagiannidis, Fellow, IEEE
}

\begin{abstract}
The $\kappa-\mu /$ inverse gamma and $\eta-\mu /$ inverse gamma composite fading models are presented and extensively investigated in this paper. We derive closed-form expressions for the fundamental statistics of the $\kappa-\mu /$ inverse gamma composite fading model, such as the probability density function (PDF), cumulative distribution function (CDF), moment generating function (MGF), higher order moments and amount of fading (AF). Closed-form expressions for the PDF, higher order moments and AF are also obtained for the $\eta-\mu /$ inverse gamma composite fading model while infinite series expressions are obtained for the corresponding CDF and MGF. The suitability of the new models for characterizing composite fading channels is demonstrated through a series of extensive field measurements for wearable, cellular and vehicular communications. For all of the measurements, two propagation geometry problems with special relevance to the two new composite fading models, namely the line-of-sight (LOS) and non-LOS (NLOS) channel conditions, are considered. It is found that both the $\kappa-\mu /$ inverse gamma and $\eta-\mu /$ inverse gamma composite fading models provide an excellent fit to fading conditions encountered in the field. The goodness-of-fit of these two composite fading models is also evaluated and compared using the resistor-average distance. As a result, it is shown that the $\kappa-\mu$ / inverse gamma composite fading model provides a better fit compared to the $\eta-\mu /$ inverse gamma composite fading model when strong dominant signal components exist. On the contrary, the $\eta-\mu /$ inverse gamma composite fading model outperforms the $\kappa-\mu /$ inverse gamma composite fading model when there is no strong dominant signal component and/or the parameter $\eta$ is not equal to unity, indicating that the scattered wave power of the in-phase and quadrature components of each cluster of multipath are not identical.
\end{abstract}

Index Terms-Channel modeling, composite fading channel, $\eta$ $\mu$ fading model, inverse gamma distribution, $\kappa-\mu$ fading model, resistor-average distance.

This work was supported in part by the U.K. Engineering and Physical Sciences Research Council under Grant No. EP/L026074/1 and the Department for the Economy Northern Ireland through Grant No. USI080. This paper was presented in part at IEEE PIMRC '15, Hong Kong, China.

S. K. Yoo, N. Bhargav, S. L. Cotton and M. Matthaiou are with the Institute of Electronics, Communications and Information Technology (ECIT), Queen's University Belfast, UK, BT3 9DT (e-mail: \{sk.yoo; nbhargav01; simon.cotton; m.matthaiou\}@qub.ac.uk)

P. C. Sofotasios is with the Department of Electrical and Computer Engineering, Khalifa University of Science and Technology, PO Box 127788, Abu Dhabi, UAE and also with the Department of Electronics and Communications Engineering, Tampere University of Technology, 33101 Tampere, Finland (email: p.sofotasios@ieee.org).

M. Valkama is with the Department of Electronics and Communications Engineering, Tampere University of Technology, 33101 Tampere, Finland (email: mikko.e.valkama@tut.fi).

G. K. Karagiannidis is with the Department of Electrical and Computer Engineering, Aristotle University of Thessaloniki, 54124, Greece (e-mail: geokarag@auth.gr).

\section{INTRODUCTION}

$\mathbf{I}$ $\mathrm{N}$ wireless communications, fading mainly occurs due to the interaction of signal components generated by multipath and shadowing phenomena. In reality, both multipath and shadowing co-exist and affect the wireless communications channel simultaneously, causing the random fluctuation of the received signal which can deteriorate the quality of radio links. Therefore, it is crucial to characterize fading behavior accurately in order to analyze wireless systems and improve their performance [1]. To this end, a number of studies have proposed the use of composite fading models, also called shadowed fading models, for both conventional and emerging communications channels. The main advantage of using composite fading models is that they provide means for more realistic channel modeling as they take into account the simultaneous impact of multipath and shadowing. Another advantage is that they circumvent the requirement to determine an appropriate smoothing window size for the computation of the local mean signal which can fundamentally affect the parameter estimation process and any inference made from the channel data. Existing composite fading models can be broadly divided into two different types according to the condition of shadowing. The first one is line-of-sight (LOS) shadowing, where the dominant signal component of the envelope is shadowed, whereas the second one is multiplicative shadowing in which the total power of the dominant (if present) and scattered signal components are shadowed [2].

Traditionally, several composite fading models have been developed based on classical fading models such as Rayleigh, Rice (Nakagami- $n$ ) and Nakagami- $m$, in which either the $L O S$ or multiplicative shadowing is assumed to follow the lognormal distribution [3]-[7]. However, the mathematical form of the lognormal distribution renders it relatively intractable for the analytical calculations associated with the performance evaluation of communications systems. This is largely based on the fact that the probability density functions (PDFs) of the lognormal-based composite fading models involve an infinite integral, which restricts the derivation of tractable analytical expressions for the performance measures of interest.

Due to the aforementioned intractability of the lognormal distribution, the gamma distribution, which can exhibit adequate semi-heavy-tailed behavior, has been proposed as an alternative to the lognormal distribution [8]. The use of the 
gamma distribution has led to closed-form expressions for the PDFs of a number of composite fading models [9], [10]. For example, in [9], the Nakagami-m / gamma composite fading model, also known as the generalized $K$ distribution $\left(K_{G}\right)$ was proposed and closed-form expressions for its PDF, outage probability and average BER for the differential phase shift keying (DPSK) modulation scheme were derived. As alternative to the lognormal distribution, the inverse Gaussian distribution, also known as the Wald distribution, has been utilized due to its ability to closely approximate the lognormal distribution [11]. Using the inverse Gaussian distribution, closed-form expressions for a set of composite fading models have been obtained in [12]-[14]. For example, in [12], the Rayleigh / inverse Gaussian composite fading model was obtained while in [14], the Nakagami- $m$ / inverse Gaussian model, which is also referred to as the $G$ distribution, was proposed.

More recently, there have been a number of studies [15][22] which have proposed the use of the more general fading models such as $\kappa-\mu, \alpha-\mu$ and $\eta-\mu$ to describe the envelope fluctuations. Subsequently, the authors of [15], [16] proposed the $\kappa-\mu /$ inverse Gaussian and $\eta-\mu /$ inverse Gaussian composite fading models, respectively, and derived expressions for their PDFs using exact infinite series expansions. In [17], the $\kappa-\mu /$ lognormal shadowed fading model was derived under the assumption that the scattered components are subject to $\kappa-\mu$ fading and the resultant dominant component is shadowed and lognormally distributed. Unfortunately, it was not possible to obtain a closed-form expression for the PDF and thus it has to be computed numerically. The author of [18] proposed the $\kappa-\mu$ shadowed fading model which assumes that the dominant component is weighted by a Nakagami- $m$ random variable (RV). Based on this, closed-form expressions for the corresponding PDF, cumulative distribution function (CDF) and moment generating function (MGF) were provided. In [19], the $\kappa-\mu$ / gamma composite fading model was proposed, which assumes that the mean signal power of a $\kappa-\mu$ fading signal varies according to the gamma distribution. Here, due to the inherent mathematical complexity of the resulting integral, the derivation of a closed-form expression for the corresponding PDF was infeasible. Instead, an approximation was provided using an infinite series expansion. Additionally, in [20], the $\kappa-\mu /$ gamma model was empirically validated using wearable off-body channel measurements conducted in indoor environments. In the same context, an approximation for the PDF of the $\eta-\mu$ / gamma composite fading model was provided in [21] whereas a closed-form expression was derived in [22]. However, the resulting formulation is only valid for integer values of the $\mu$ parameter [22].

As an alternative, the $\kappa-\mu /$ inverse gamma [23] and $\eta$ $\mu$ / inverse gamma [24] models have been proposed by considering the use of the inverse gamma distribution for characterizing shadowing ${ }^{1}$. As shown in Table I, they are extremely flexible models which contain as special cases the

\footnotetext{
${ }^{1}$ Similar to the lognormal and gamma distributions, the inverse gamma distribution can also exhibit the semi heavy-tailed characteristics necessary to accurately characterize shadowing. As well as this, it also offers much of the analytical tractability available from using the gamma distribution.
}

many of the existing fading models proposed in the open literature. In these studies, the PDFs of both models have been presented in simple closed-form expressions and were shown to exhibit great promise for modeling the composite fading signal observed in wearable communications channels. Motivated by these results, we extend this empirical validation to include a diverse range of emerging wireless applications, such as wearable, cellular and vehicular communications. Furthermore, we provide additional physical and technical insights into the properties of the proposed composite fading models, as well as deriving the fundamental statistics of the $\kappa$ $\mu$ / inverse gamma and $\eta-\mu /$ inverse gamma composite fading models. Accordingly, the main contributions of this paper can be summarized as follows:

- We derive novel, closed-form expressions for the PDF, CDF, MGF, higher order moments and amount of fading (AF) of the $\kappa-\mu /$ inverse gamma composite fading model.

- We derive the PDF, higher order moments and AF of the $\eta-\mu /$ inverse gamma composite fading model in closed-form while infinite series representations, that are shown to be analytically convergent, are obtained for the corresponding CDF and MGF.

- These fundamental statistics are essential for the accurate characterization of fading channels, as well as for the computation of several communications performance metrics of interest.

- The generality of the $\kappa-\mu /$ inverse gamma and $\eta-\mu /$ inverse gamma models is highlighted through reduction to some special cases which coincide with existing wellknown distributions, as well as their ability to approximate other composite fading models commonly encountered in the literature.

- An important empirical validation of the $\kappa-\mu /$ inverse gamma and $\eta-\mu$ / inverse gamma models is demonstrated using field measurements for three different emerging wireless applications, namely wearable, cellular and vehicular communications. The goodness-of-fit of the proposed models is also evaluated using the resistor-average distance (RAD).

The remainder of this paper is organized as follows: In Section II, we introduce the fundamental statistics of the $\kappa$ $\mu /$ inverse gamma and $\eta-\mu /$ inverse gamma models. Then, the utility of the proposed models is validated using a diverse range of field measurements in Sections III, IV and V. Finally, Section VI concludes the paper with some closing remarks.

\section{The New Composite FAding Models}

\section{A. $\kappa-\mu /$ Inverse Gamma Composite Fading Model}

Similar to the physical signal model proposed for the $\kappa$ $\mu$ fading channel [25], the received signal in a $\kappa-\mu /$ inverse gamma composite fading channel is composed of separable clusters of multipath waves propagating in a homogeneous environment. The power of the scattered waves from the multipath clusters is assumed to be identical whereas the power of the dominant wave within each cluster is assumed to be arbitrary. Unlike the $\kappa-\mu$ fading channel, however, in 
TABLE I

Special Cases of the $\kappa-\mu$ / Inverse Gamma And $\eta-\mu$ / Inverse Gamma Composite Fading Models

\begin{tabular}{|c|c|c|}
\hline & $\begin{array}{c}\kappa-\mu / \text { inverse gamma model } \\
\left(\kappa, \mu, m_{s}, \Omega\right)\end{array}$ & $\begin{array}{c}\eta-\mu / \text { inverse gamma model } \\
\left(\eta, \mu, m_{s}, \Omega\right)\end{array}$ \\
\hline One-sided Gaussian $(\Omega)$ & $\kappa \rightarrow 0, \mu=0.5, m_{s} \rightarrow \infty, \Omega=\Omega$ & $\begin{array}{c}\eta \rightarrow 0 \text { (or } \eta \rightarrow \infty), \mu=0.5, m_{s} \rightarrow \infty, \Omega=\Omega \text { or } \\
\eta \rightarrow 1, \mu=0.5 / 2, m_{s} \rightarrow \infty, \Omega=\Omega\end{array}$ \\
\hline Rayleigh $(\Omega)$ & $\kappa \rightarrow 0, \mu=1, m_{s} \rightarrow \infty, \Omega=\Omega$ & $\begin{array}{c}\eta \rightarrow 0 \text { (or } \eta \rightarrow \infty), \mu=1, m_{s} \rightarrow \infty, \Omega=\Omega \text { or } \\
\eta \rightarrow 1, \mu=1 / 2, m_{s} \rightarrow \infty, \Omega=\Omega\end{array}$ \\
\hline Rice $(K, \Omega)$ & $\kappa=K, \mu=1, m_{s} \rightarrow \infty, \Omega=\Omega$ & - \\
\hline Nakagami- $m(m, \Omega)$ & $\kappa \rightarrow 0, \mu=m, m_{s} \rightarrow \infty, \Omega=\Omega$ & $\begin{array}{c}\eta \rightarrow 0 \text { (or } \eta \rightarrow \infty), \mu=m_{s} \rightarrow \infty, \Omega=\Omega \text { or } \\
\eta \rightarrow 1, \mu=m / 2, m_{s} \rightarrow \infty, \Omega=\Omega\end{array}$ \\
\hline Nakagami- $q(q, \Omega)$ & - & $\eta=q^{2}, \mu=0.5, m_{s} \rightarrow \infty, \Omega=\Omega$ \\
\hline$\kappa-\mu(\kappa, \mu, \Omega)$ & $\kappa=\kappa, \mu=\mu, m_{s} \rightarrow \infty, \Omega=\Omega$ & - \\
\hline$\eta-\mu(\eta, \mu, \Omega)$ & - & $\eta=\eta, \mu=\mu, m_{s} \rightarrow \infty, \Omega=\Omega$ \\
\hline
\end{tabular}

$\dagger$ In this paper, Format 1 is adopted to represent the $\eta$ - $\mu$ / inverse gamma composite fading model. However, Format 2 can be also deduced straightforwardly.

the $\kappa-\mu /$ inverse gamma fading channel, the mean power of the multipath waves (i.e. both the dominant and scattered waves) is randomly fluctuated due to shadowing. Therefore, the composite signal envelope, $R$, in a $\kappa-\mu$ / inverse gamma composite fading channel can be expressed in terms of the in-phase and quadrature components as

$$
R=\sqrt{\sum_{i=1}^{n_{s}} A\left(I_{i}+p_{i}\right)^{2}+A\left(Q_{i}+q_{i}\right)^{2}}
$$

where $n_{s}$ represents the number of clusters of multipath, $I_{i}$ and $Q_{i}$ are mutually independent Gaussian RVs with $\mathbb{E}\left[I_{i}\right]=$ $\mathbb{E}\left[Q_{i}\right]=0$ and $\mathbb{E}\left[I_{i}^{2}\right]=\mathbb{E}\left[Q_{i}^{2}\right]=\sigma^{2}$, with $\mathbb{E}[\cdot]$ denoting statistical expectation, while $p_{i}$ and $q_{i}$ are the mean values of the in-phase and quadrature components of the multipath cluster $i$, respectively. In (1), $A$ denotes an inverse gamma RV with the shape parameter $m_{s}$ and scale parameter $m_{s} / \Omega_{s}$, where $\Omega_{s}$ is set equal to unity (i.e. $\Omega_{s}=1$ ). To this effect, the PDF of $A$ can be written as follows

$$
f_{A}(\alpha)=\frac{m_{s}{ }^{m_{s}}}{\Gamma\left(m_{s}\right) \alpha^{m_{s}+1}} \exp \left(-\frac{m_{s}}{\alpha}\right)
$$

where $\Gamma(\cdot)$ denotes the gamma function [26, Eq. (8.310.1)].

Theorem 1. For $\kappa, \mu, m_{s}, \Omega, r \in \mathbb{R}^{+}$, the PDF of the composite signal envelope in a $\kappa-\mu /$ inverse gamma composite fading channel can be expressed as

$$
\begin{aligned}
f_{R}(r)= & \frac{\exp (-\mu \kappa) 2 \mu^{\mu}(\kappa+1)^{\mu}\left(m_{s} \Omega\right)^{m_{s}} r^{2 \mu-1}}{B\left(m_{s}, \mu\right)\left[\mu(\kappa+1) r^{2}+m_{s} \Omega\right]^{m_{s}+\mu}} \\
& \times_{1} F_{1}\left(m_{s}+\mu ; \mu ; \frac{\mu^{2} \kappa(\kappa+1) r^{2}}{\mu(\kappa+1) r^{2}+m_{s} \Omega}\right)
\end{aligned}
$$

where $B(\cdot, \cdot)$ and ${ }_{1} F_{1}(\cdot ; \cdot ; \cdot)$ denote the Beta function [26, Eq. (8.384.1)] and the Kummer confluent hypergeometric function [26, Eq. (9.210.1)], respectively.

Proof: See Appendix A-A.

Remark 1. In terms of its physical interpretation, $\kappa>0$ is the ratio of the total power of the dominant components $\left(\delta^{2}=\sum_{i=1}^{n_{s}} p_{i}^{2}+q_{i}^{2}\right)$ to the total power of the scattered waves $\left(2 \mu \sigma^{2}\right)$, while $\mu$ is related to the number of multipath clusters, with $2 \sigma^{2}$ denoting the power of the scattered waves in each cluster. In this model, the mean signal power is given by $\mathbb{E}\left[R^{2}\right]=\Omega=\delta^{2}+2 \mu \sigma^{2}$.

The corresponding PDF of the instantaneous SNR of the $\kappa$ $\mu$ / inverse gamma composite fading model is also readily obtained by letting $\gamma=\bar{\gamma} r^{2} / \Omega$, where $\bar{\gamma}=\mathbb{E}[\gamma]$, such that

$$
\begin{aligned}
f_{\gamma}(\gamma)= & \frac{\exp (-\mu \kappa) \mu^{\mu}(\kappa+1)^{\mu}\left(m_{s} \bar{\gamma}\right)^{m_{s}} \gamma^{\mu-1}}{B\left(m_{s}, \mu\right)\left[\mu(\kappa+1) \gamma+m_{s} \bar{\gamma}\right]_{s}^{m_{s}+\mu}} \\
& \times{ }_{1} F_{1}\left(m_{s}+\mu ; \mu ; \frac{\mu^{2} \kappa(\kappa+1) \gamma}{\mu(\kappa+1) \gamma+m_{s} \bar{\gamma}}\right) .
\end{aligned}
$$

It is worth highlighting that the resultant PDF in (4) is functionally equivalent to the singly non-central $\mathcal{F}$ distribution ${ }^{2}$ that arises as a result of the ratio of a non-central chi-squared variable and a central chi-squared variable [28].

Theorem 2. For $\kappa, \mu, m_{s}, \gamma, \bar{\gamma} \in \mathbb{R}^{+}$, the CDF of the instantaneous SNR of the $\kappa-\mu /$ inverse gamma model can be obtained as

$$
\begin{aligned}
& F_{\gamma}(\gamma)=\sum_{i=0}^{\infty} \frac{\exp (-\mu \kappa)(\mu \kappa)^{i}}{i !(\mu+i) B\left(m_{s}, \mu+i\right)}\left(\frac{\mu(\kappa+1) \gamma}{m_{s} \bar{\gamma}}\right)^{\mu+i} \\
& \quad \times_{2} F_{1}\left(m_{s}+\mu+i, \mu+i ; \mu+i+1 ;-\frac{\mu(\kappa+1) \gamma}{m_{s} \bar{\gamma}}\right)
\end{aligned}
$$

where ${ }_{2} F_{1}(\cdot, \cdot ; \cdot ; \cdot)$ denotes the Gauss hypergeometric function [26, Eq. (9.111)]. For the case of $m_{s} \bar{\gamma}>\mu(\kappa+1) \gamma$, (5) can be rewritten in closed-form as follows

$$
\begin{aligned}
& F_{\gamma}(\gamma)=\frac{\exp (-\mu \kappa)}{\mu B\left(m_{s}, \mu\right)}\left(\frac{\mu(\kappa+1) \gamma}{m_{s} \bar{\gamma}}\right)^{\mu} \\
& \times F_{1,1,0}^{2,0,0}\left(\begin{array}{c}
m_{s}+\mu, \mu ;-;-; \mu^{2} \kappa(\kappa+1) \gamma \\
\mu+1 ; \mu ;-;
\end{array} m_{s} \bar{\gamma},-\frac{\mu(\kappa+1) \gamma}{m_{s} \bar{\gamma}}\right)
\end{aligned}
$$

where

$$
F_{\mathcal{P}, \mathcal{Q}, \mathcal{S}}^{\mathcal{A}, \mathcal{B}}\left(\begin{array}{l}
a_{1}, \ldots, a_{\mathcal{A}} ; b_{1}, \ldots, b_{\mathcal{B}} ; c_{1}, \ldots, c_{\mathcal{C}} ; \\
p_{1}, \ldots, p_{\mathcal{P}} ; q_{1}, \ldots, q_{\mathcal{Q}} ; s_{1}, \ldots, s_{\mathcal{S}}
\end{array} ; \cdot\right)
$$

represents the Kampé de Fériet function [29]. On the contrary,

${ }^{2}$ Using [27, Eq. (2.312)], i.e. $f_{X}(x)=\bar{\gamma} f_{\gamma}(\bar{\gamma} \gamma)$, letting $\gamma(1+\kappa)=x$ and then performing the requisite transformation along with the following substitutions $\mu=d_{1} / 2, m_{s}=d_{2} / 2, \mu \kappa=\lambda / 2$, we can obtain the singly non-central $\mathcal{F}$ distribution, $f_{x}(x)$, with parameters $d_{1}, d_{2}$ and $\lambda$. 
for the case of $m_{s} \bar{\gamma} \leq \mu(\kappa+1) \gamma, F_{\gamma}(\gamma)$ can be expressed in closed-form as

$$
\begin{aligned}
& F_{\gamma}(\gamma)=\exp (-\mu \kappa) \Psi_{1}\left(\mu ; 0 ; 1-m_{s}, \mu,-\frac{m_{s} \bar{\gamma}}{\mu(\kappa+1) \gamma}, \mu \kappa\right) \\
& -\left[\frac{\exp (-\mu \kappa)}{m_{s} B\left(m_{s}, \mu\right)}\left(\frac{m_{s} \bar{\gamma}}{\mu(\kappa+1) \gamma}\right)^{m_{s}}\right. \\
& \left.\quad \times \Psi_{1}\left(m_{s}+\mu ; m_{s} ; 1+m_{s}, \mu,-\frac{m_{s} \bar{\gamma}}{\mu(\kappa+1) \gamma}, \mu \kappa\right)\right]
\end{aligned}
$$

where $\Psi_{1}(\cdot ; \cdot ; \cdot, \cdot, \cdot, \cdot)$ denotes the Humbert $\Psi_{1}$ function [30].

Proof: See Appendix A-B.

Theorem 3. For $\kappa, \mu, m_{s}, \bar{\gamma} \in \mathbb{R}^{+}$, the higher order moments of the instantaneous SNR of the $\kappa-\mu /$ inverse gamma model can be expressed as

$$
\mathbb{E}\left[\gamma^{n}\right]=\left(\frac{m_{s} \bar{\gamma}}{\mu(\kappa+1)}\right)^{n} \frac{B\left(m_{s}-n, \mu+n\right)_{1} F_{1}(\mu+n ; \mu ; \mu \kappa)}{\exp (\mu \kappa) B\left(m_{s}, \mu\right)} .
$$

Proof: See Appendix A-C.

It is recalled here that the AF is often used as a relative measure of the severity of fading encountered in wireless transmission over fading channels.

Corollary 1. For $\kappa, \mu, m_{s} \in \mathbb{R}^{+}$, the $A F$ for the case of a $\kappa-\mu /$ inverse gamma composite fading channel is given by

$$
\mathrm{AF}=\frac{\mu(\kappa+1)^{2}+\left(m_{s}-1\right)(2 \kappa+1)}{\mu(\kappa+1)^{2}\left(m_{s}-2\right)} .
$$

Proof: See Appendix A-D.

Theorem 4. For $\kappa, \mu, m_{s}, \bar{\gamma}, s \in \mathbb{R}^{+}, m_{s}+\mu+i \neq \mathbb{N}$ and $m_{s} \neq \mathbb{N}$, the MGF of the $\kappa-\mu /$ inverse gamma distribution can be expressed as follows

$$
\begin{aligned}
M_{\gamma}(-s) & =\exp (-\mu \kappa) \Psi_{2}\left(\mu ; \mu, 1-m_{s}, \mu \kappa, \frac{s m_{s} \bar{\gamma}}{\mu(\kappa+1)}\right) \\
+ & {\left[\frac{\exp (-\mu \kappa)}{B\left(m_{s}, \mu\right)}\left(\frac{s m_{s} \bar{\gamma}}{\mu(\kappa+1)}\right)^{m_{s}} \Gamma\left(-m_{s}\right)\right.} \\
& \left.\times \Psi_{2}\left(m_{s}+\mu ; \mu, 1+m_{s}, \mu \kappa, \frac{s m_{s} \bar{\gamma}}{\mu(\kappa+1)}\right)\right]
\end{aligned}
$$

where $\Psi_{2}(\cdot ; \cdot, \cdot, \cdot, \cdot)$ denotes the Humbert $\Psi_{2}$ function [30].

Proof: See Appendix A-E.

\section{B. $\eta-\mu$ / Inverse Gamma Composite Fading Model}

Similar to the physical signal model proposed for the $\eta-\mu$ fading channel [25], the received signal in an $\eta-\mu /$ inverse gamma composite fading channel is composed of separable clusters of multipath waves propagating in a non-homogeneous environment. In Format 1, the in-phase and quadrature components of the fading signal within each cluster are assumed to be statistically independent from each other and to have different power. On the other hand, in Format 2, the in-phase and quadrature components of the fading signal within each cluster are assumed to be correlated with each other and to have identical power. Unlike the $\eta-\mu$ fading model, in the $\eta$ $\mu /$ inverse gamma model, the mean power of the scattered waves is randomly fluctuated due to shadowing. Following this definition, the composite signal envelope, $R$, in an $\eta-\mu /$ inverse gamma composite fading channel can be expressed as

$$
R=\sqrt{\sum_{i=1}^{n_{s}} A I_{i}^{2}+A Q_{i}^{2}}
$$

where $n_{s}$ denotes the number of clusters of multipath and $A$ represents an inverse gamma RV given in (2). In Format $1, I_{i}$ and $Q_{i}$ are mutually independent Gaussian RVs with $\mathbb{E}\left[I_{i}\right]=\mathbb{E}\left[Q_{i}\right]=0, \mathbb{E}\left[I_{i}^{2}\right]=\sigma_{I}^{2}$ and $\mathbb{E}\left[Q_{i}^{2}\right]=\sigma_{Q}^{2}$, while in Format $2, I_{i}$ and $Q_{i}$ are mutually correlated Gaussian RVs with $\mathbb{E}\left[I_{i}\right]=\mathbb{E}\left[Q_{i}\right]=0$, and $\mathbb{E}\left[I_{i}^{2}\right]=\mathbb{E}\left[Q_{i}^{2}\right]=\sigma^{2}$. In what follows, we derive the PDF and CDF of the $\eta-\mu /$ inverse gamma composite fading model.

Theorem 5. For $\eta, \mu, m_{s}, \Omega, r \in \mathbb{R}^{+}$, the PDF of the composite signal envelope in an $\eta-\mu /$ inverse gamma composite fading channel can be expressed as

$$
\begin{aligned}
& f_{R}(r)=\frac{2^{2 \mu+1} \mu^{2 \mu} h^{\mu}\left(m_{s} \Omega\right)^{m_{s}} r^{4 \mu-1}}{B\left(m_{s}, 2 \mu\right)\left(2 \mu h r^{2}+m_{s} \Omega\right)^{m_{s}+2 \mu}} \\
& \times_{2} F_{1}\left(\frac{m_{s}+2 \mu}{2}, \frac{m_{s}+2 \mu+1}{2} ; \frac{2 \mu+1}{2} ; \frac{\left(2 \mu H r^{2}\right)^{2}}{\left(2 \mu h r^{2}+m_{s} \Omega\right)^{2}}\right) .
\end{aligned}
$$

Proof: See Appendix B-A.

Remark 2. In terms of its physical interpretation, $\eta$ is defined as $\eta=\sigma_{I}^{2} / \sigma_{Q}^{2}$ (i.e. the scattered wave power ratio between the in-phase and quadrature components of each cluster of multipath) in Format 1 , whereas $\eta=$ $\mathbb{E}\left[I_{i} Q_{i}\right] / \sigma^{2}$ (i.e. the correlation coefficient between the inphase and quadrature components) in Format 2. Accordingly, $h=\left(2+\eta^{-1}+\eta\right) / 4$ and $H=\left(\eta^{-1}-\eta\right) / 4$ in Format 1 , while $h=1 /\left(1-\eta^{2}\right)$ and $H=\eta /\left(1-\eta^{2}\right)$ in Format 2. Based on this, Format 1 can be obtained from Format 2 and vice versa by using the following relationship $\eta_{\text {Format } 1}=\left(1-\eta_{\text {Format } 2}\right) /\left(1+\eta_{\text {Format } 2}\right)$ or, equivalently by $\eta_{\text {Format } 2}=\left(1-\eta_{\text {Format } 1}\right) /\left(1+\eta_{\text {Format } 1}\right)$, where $0<$ $\eta_{\text {Format } 1}<\infty$ in Format 1 and $-1<\eta_{\text {Format } 2}<1$ in Format 2. In this model, the mean signal power is given by $\mathbb{E}\left[R^{2}\right]=\Omega=\mu\left(1+\eta^{-1}\right) \sigma_{I}^{2}=\mu(1+\eta) \sigma_{Q}^{2}$ in Format 1 whereas it is given by $\mathbb{E}\left[R^{2}\right]=\Omega=2 \mu \sigma^{2}$ in Format 2 .

Based on the above, the PDF of the instantaneous SNR of the $\eta-\mu /$ inverse gamma composite fading model can be easily expressed with the aid of $\gamma=\bar{\gamma} r^{2} / \Omega$ as follows

$$
\begin{aligned}
& f_{\gamma}(\gamma)=\frac{2^{2 \mu} \mu^{2 \mu} h^{\mu}\left(m_{s} \bar{\gamma}\right)^{m_{s}} \gamma^{2 \mu-1}}{B\left(m_{s}, 2 \mu\right)\left(2 \mu h \gamma+m_{s} \bar{\gamma}\right)^{m_{s}+2 \mu}} \\
& \times_{2} F_{1}\left(\frac{m_{s}+2 \mu}{2}, \frac{m_{s}+2 \mu+1}{2} ; \frac{2 \mu+1}{2} ; \frac{(2 \mu H \gamma)^{2}}{\left(2 \mu h \gamma+m_{s} \bar{\gamma}\right)^{2}}\right) .
\end{aligned}
$$

Theorem 6. For $\eta, \mu, m_{s}, \gamma, \bar{\gamma} \in \mathbb{R}^{+}$, the CDF of the instantaneous SNR of the $\eta-\mu /$ inverse gamma model can be obtained as follows

$$
\begin{aligned}
F_{\gamma}(\gamma) & =\frac{2^{2 \mu-1} h^{\mu}}{\Gamma\left(m_{s}\right) \Gamma(2 \mu)} \sum_{i=0}^{\infty} \frac{\Gamma\left(m_{s}+2 \mu+2 i\right) H^{2 i}}{i !\left(\frac{2 \mu+1}{2}\right)_{i}(\mu+i)}\left(\frac{\mu \gamma}{m_{s} \bar{\gamma}}\right)^{2 \mu+2 i} \\
& \times{ }_{2} F_{1}\left(m_{s}+2 \mu+2 i, 2 \mu+2 i ; 2 \mu+2 i+1 ;-\frac{2 \mu h \gamma}{m_{s} \bar{\gamma}}\right)
\end{aligned}
$$


where $(\cdot)_{i}$ denotes the Pochhammer symbol [31, Eq. (06.10.02.0001.01)].

Proof: See Appendix B-B.

The truncation error, $\mathcal{T}$, for the infinite series in (14) if $T_{0}-1$ terms are used is expressed as

$$
\begin{aligned}
& \mathcal{T}=\sum_{i=T_{0}}^{\infty} \frac{\Gamma\left(m_{s}+2 \mu+2 i\right) H^{2 i}}{i !\left(\frac{2 \mu+1}{2}\right)_{i}(\mu+i)}\left(\frac{\mu \gamma}{m_{s} \bar{\gamma}}\right)^{2 \mu+2 i} \\
& \quad \times{ }_{2} F_{1}\left(m_{s}+2 \mu+2 i, 2 \mu+2 i ; 2 \mu+2 i+1 ;-\frac{2 \mu h \gamma}{m_{s} \bar{\gamma}}\right) .
\end{aligned}
$$

Since the Gauss hypergeometric function in (15) is monotonically decreasing with respect to $i, \mathcal{T}$ can be bounded as

$$
\begin{aligned}
\mathcal{T} \leq & { }_{2} F_{1}\left(m_{s}+2 \mu+2 T_{0}, 2 \mu+2 T_{0} ; 2 \mu+2 T_{0}+1 ;-\frac{2 \mu h \gamma}{m_{s} \bar{\gamma}}\right) \\
& \times \sum_{i=T_{0}}^{\infty} \frac{\Gamma\left(m_{s}+2 \mu+2 i\right) H^{2 i}}{i !\left(\frac{2 \mu+1}{2}\right)_{i}(\mu+i)}\left(\frac{\mu \gamma}{m_{s} \bar{\gamma}}\right)^{2 \mu+2 i} .
\end{aligned}
$$

Since we add up strictly positive terms, the summation limits in (16) can be re-written as

$\sum_{i=T_{0}}^{\infty} \frac{\Gamma\left(m_{s}+2 \mu+2 i\right)}{i !\left(\frac{2 \mu+1}{2}\right)_{i}}\left(\frac{\mu \gamma H}{m_{s} \bar{\gamma}}\right)^{2 i} \leq \sum_{i=0}^{\infty} \frac{\Gamma\left(m_{s}+2 \mu+2 i\right)}{\left(\frac{2 \mu+1}{2}\right)_{i}}\left(\frac{\mu \gamma H}{m_{s} \bar{\gamma}}\right)^{2 i}$.

For the case of $m_{s} \bar{\gamma}>2 \mu \gamma H$, using the Legendre duplication formula [31, Eq. (06.05.16.0006.01)] on $\Gamma\left(m_{s}+2 \mu+2 i\right)$, simplifying the resultant expression and then using [31, Eq. (07.23.02.0001.01)], (16) can be expressed in closed-form as

$$
\begin{aligned}
\mathcal{T} \leq & { }_{2} F_{1}\left(m_{s}+2 \mu+2 T_{0}, 2 \mu+2 T_{0} ; 2 \mu+2 T_{0}+1 ;-\frac{2 \mu h \gamma}{m_{s} \bar{\gamma}}\right)(\mu \gamma)^{2 \mu} \\
& \times \Gamma\left(m_{s}+2 \mu\right)_{2} F_{1}\left(\frac{m_{s}+2 \mu}{2}, \frac{m_{s}+2 \mu+1}{2} ; \frac{2 \mu+1}{2} ;\left(\frac{2 \mu \gamma H}{m_{s} \bar{\gamma}}\right)^{2}\right) .
\end{aligned}
$$

It is worth remarking that similar conditions (i.e. $m_{s} \bar{\gamma}>$ $2 \mu \gamma H$ ) for the convergence of the infinite series in (16) can be drawn by computing the corresponding convergence ratio.

Theorem 7. For $\eta, \mu, m_{s}, \bar{\gamma} \in \mathbb{R}^{+}$, the higher order moments of the instantaneous SNR of the proposed $\eta$ - $\mu$ / inverse gamma composite fading model can be expressed as

$$
\begin{aligned}
\mathbb{E}\left[\gamma^{n}\right] & =\frac{B\left(m_{s}-n, 2 \mu+n\right)}{B\left(m_{s}, 2 \mu\right) h^{\mu}}\left(\frac{m_{s} \bar{\gamma}}{2 \mu h}\right)^{n} \\
& \times{ }_{2} F_{1}\left(\mu+\frac{n}{2}, \mu+\frac{n}{2}+\frac{1}{2} ; \mu+\frac{1}{2} ; \frac{H^{2}}{h^{2}}\right) .
\end{aligned}
$$

Proof: See Appendix B-C.

Corollary 2. For $\eta, \mu, m_{s} \in \mathbb{R}^{+}$, the corresponding $A F$ of the instantaneous SNR of the proposed $\eta-\mu /$ inverse gamma composite fading model can be expressed as

$$
\mathrm{AF}=\frac{\left(h^{2}-H^{2}\right)^{\mu}\left(H^{2}+2 \mu h^{2}+h^{2}\right)\left(m_{s}-1\right)}{2 \mu h^{(\mu+2)}\left(m_{s}-2\right)}-1 .
$$

Proof: See Appendix B-D.
Theorem 8. For $\eta, \mu, m_{s}, \bar{\gamma}, s \in \mathbb{R}^{+}, m_{s}+2 \mu+2 i \neq \mathbb{N}$ and $m_{s} \neq \mathbb{N}$, the $M G F$ of the $\eta-\mu /$ inverse gamma distribution can be expressed as

$$
\begin{aligned}
& M_{\gamma}(-s)=\sum_{i=0}^{\infty} \frac{\Gamma(\mu+i) H^{2 i}}{i ! \Gamma(\mu) h^{\mu+2 i}}\left[{ }_{1} F_{1}\left(2 \mu+2 i ; 1-m_{s} ; \frac{s m_{s} \bar{\gamma}}{2 \mu h}\right)\right. \\
& \left.+\left(\frac{s m_{s} \bar{\gamma}}{2 \mu h}\right)^{m_{s}} \frac{\Gamma\left(-m_{s}\right)}{B\left(m_{s}, 2 \mu+2 i\right)}{ }_{1} F_{1}\left(m_{s}+2 \mu+2 i ; 1+m_{s} ; \frac{s m_{s} \bar{\gamma}}{2 \mu h}\right)\right] .
\end{aligned}
$$

Proof: See Appendix B-E.

In a similar manner to (14), the truncation error for the infinite series in (21) can be represented by the following inequality

$$
\begin{aligned}
\mathcal{T} \leq & { }_{1} F_{1}\left(2 \mu+2 T_{0} ; 1-m_{s} ; \frac{s m_{s} \bar{\gamma}}{2 \mu h}\right){ }_{1} F_{0}\left(\mu ;-; \frac{H^{2}}{h^{2}}\right) \\
- & {\left[{ }_{1} F_{1}\left(m_{s}+2 \mu+2 T_{0} ; 1+m_{s} ; \frac{s m_{s} \bar{\gamma}}{2 \mu h}\right)\right.} \\
& \left.\times{ }_{2} F_{1}\left(\frac{m_{s}+2 \mu}{2}, \frac{m_{s}+2 \mu+1}{2} ; \frac{2 \mu+1}{2} ; \frac{H^{2}}{h^{2}}\right)\right] .
\end{aligned}
$$

It is worth highlighting that both first and second sub-series in (21) are always convergent.

\section{Numerical Results}

As shown in Table I, the $\kappa-\mu /$ inverse gamma and $\eta-\mu /$ inverse gamma models are extremely versatile as they inherit all of the generality of the $\kappa-\mu$ and $\eta-\mu$ models, respectively. In both models, the $m_{s}$ parameter controls the amount of the shadowing of the mean signal power. Thus, as $m_{s} \rightarrow 0$, the dominant component (if applicable) and scattered signal component are completely shadowed. On the contrary, as $m_{s} \rightarrow \infty$, there is no shadowing present in the channel and thus the mean signal power becomes deterministic. In this case, the $\kappa-\mu /$ inverse gamma and $\eta-\mu /$ inverse gamma models coincide with the $\kappa-\mu$ and $\eta$ - $\mu$ models respectively, as illustrated in Fig. 1. Likewise, for the $\kappa-\mu /$ inverse gamma model as shown in Fig. 1(a), by setting $\mu=1$ and again letting $m_{s} \rightarrow \infty$, the Rice fading model is deduced where $\kappa$ becomes equivalent to the Rice $K$ factor. Based on this, the Rayleigh fading model can be readily obtained by setting $\kappa=K=0$. Similarly, the Nakagami- $m$ fading model can be obtained by letting $m_{s} \rightarrow \infty$ and $\kappa \rightarrow 0$ with the $\mu$ parameter becoming equivalent to the $m$ parameter of Nakagami- $m$ fading model. For the $\eta-\mu /$ inverse gamma model as shown in Fig. 1(b), by setting $\mu=0.5$ and again letting $m_{s} \rightarrow \infty$, the Nakagami- $q$ (or Hoyt) fading model is deduced where $\eta$ becomes equivalent to the square of the $q$ parameter of Nakagami- $q$ fading model (i.e. $\eta=q^{2}$ ). The Nakagami- $m$ fading model can be obtained by letting $m_{s} \rightarrow \infty$ and $\eta \rightarrow 0$ with the $\mu$ parameter becoming equivalent to the $m$ parameter of Nakagami- $m$ fading model. Based on this, the Rayleigh fading model can also be readily obtained by setting $\eta=m=1$. It should be noted that Format 1 is utilized for the $\eta-\mu /$ inverse gamma composite fading model, however extensions to the case of Format 2 are straightforward.

It is worth highlighting that they can be also used to provide an accurate approximation of other lognormal- and gamma- 

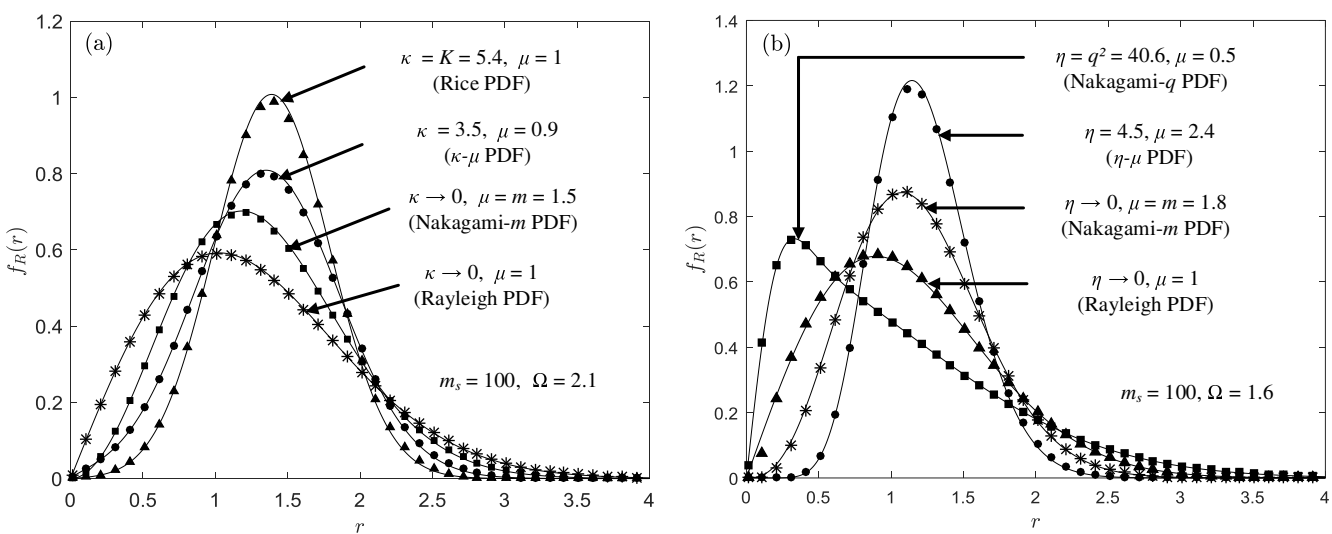

Fig. 1. PDFs of (a) the $\kappa-\mu$ / inverse gamma composite model (continuous lines) for the special cases: $\kappa-\mu$ (circles), Nakagami- $m$ (squares), Rice (triangles) and Rayleigh (asterisks) fading models; (b) the $\eta-\mu$ / inverse gamma composite model (continuous lines) for the special cases: $\eta-\mu$ (circles), Nakagami- $q$ (squares), Rayleigh (triangles) and Nakagami- $m$ (asterisks) fading models.
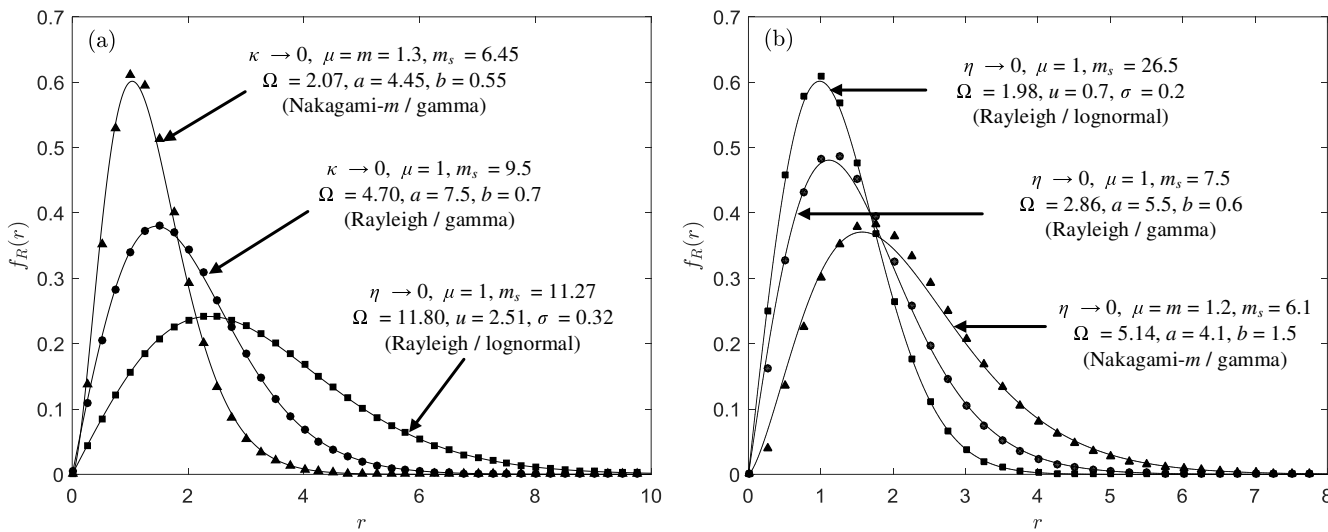

Fig. 2. PDFs of (a) the $\kappa-\mu$ / inverse gamma composite fading model (continuous lines) and (b) the $\eta-\mu /$ inverse gamma composite fading model (continuous lines) for the approximation of the Rayleigh / gamma (circles), Rayleigh / lognormal (squares) and Nakagami-m / gamma (triangles) composite fading models.

based composite fading models. For example, Fig. 2 shows that both the $\kappa-\mu /$ inverse gamma $\left(\kappa, \mu, m_{s}, \Omega\right)$ and $\eta$ $\mu /$ inverse gamma $\left(\eta, \mu, m_{s}, \Omega\right)$ composite fading models provide a good match to the Rayleigh / lognormal $(u, \sigma)$ [5], Rayleigh / gamma $(a, b)[10]$ and Nakagami- $m$ / gamma ( $m$, $a, b)$ [9] composite fading models. For this comparison, the $m_{s}$ and $\Omega$ parameters of the $\kappa-\mu /$ inverse gamma and $\eta-\mu /$ inverse gamma composite fading models were estimated from the $u$ and $\sigma$ parameters of the Rayleigh / lognormal model and from the $a$ and $b$ parameters of the Rayleigh / gamma and Nakagami- $m$ / gamma models by matching their first and second moments, such that

$$
\begin{aligned}
& m_{s}=\frac{2 \exp \left(\sigma^{2}\right)-1}{\exp \left(\sigma^{2}\right)-1}, \\
& \Omega=\frac{\exp \left(u+\frac{3}{2} \sigma^{2}\right)}{m_{s}\left(\exp \left(\sigma^{2}\right)-1\right)}
\end{aligned}
$$

and

$$
\begin{gathered}
m_{s}=a+2, \\
\Omega=\frac{a b(a+1)}{m_{s}} .
\end{gathered}
$$

Fig. 3 shows the estimated AF values for different values of the respective parameters of the $\kappa-\mu /$ inverse gamma and $\eta-\mu /$ inverse gamma composite fading models, i.e. $0 \leq \kappa \leq$ $10,0<\eta \leq 10,0 \leq \mu \leq 10$ and $3 \leq m_{s} \leq 20$. It is clear that for the $\kappa-\mu /$ inverse gamma model the greatest AF occurs when the channel is subject to simultaneous heavy shadowing $\left(m_{s} \rightarrow 3\right)$, no dominant signal component $(\kappa \rightarrow 0)$ and fewer numbers of multipath clusters $(\mu \rightarrow 0)$. On the contrary, the value of the AF approaches zero as the $\kappa, \mu$ and $m_{s}$ parameters become large. For the $\eta-\mu /$ inverse gamma model, large AFs appear when there exists heavy shadowing $\left(m_{s} \rightarrow 3\right)$, a lesser number of multipath clusters $(\mu \rightarrow 0)$ and there is a difference between scattered wave power of in-phase and quadrature components (i.e. $\eta \neq 1$ ).

\section{Application I - Wearable Communications CHANNELS}

In the sequel, we demonstrate the practical application of the two novel fading models proposed in the previous section. We begin with the emerging area of wearable communications which have recently received significant attention due to the wide range of promising application areas including medical, sports, military and entertainment [32]-[35]. In this study, we consider wearable off-body channels which are an important part of personal area networks (PANs). In wearable PAN applications, one or more wireless devices on the human body typically communicate with transceivers or base stations situated in the local surroundings. 

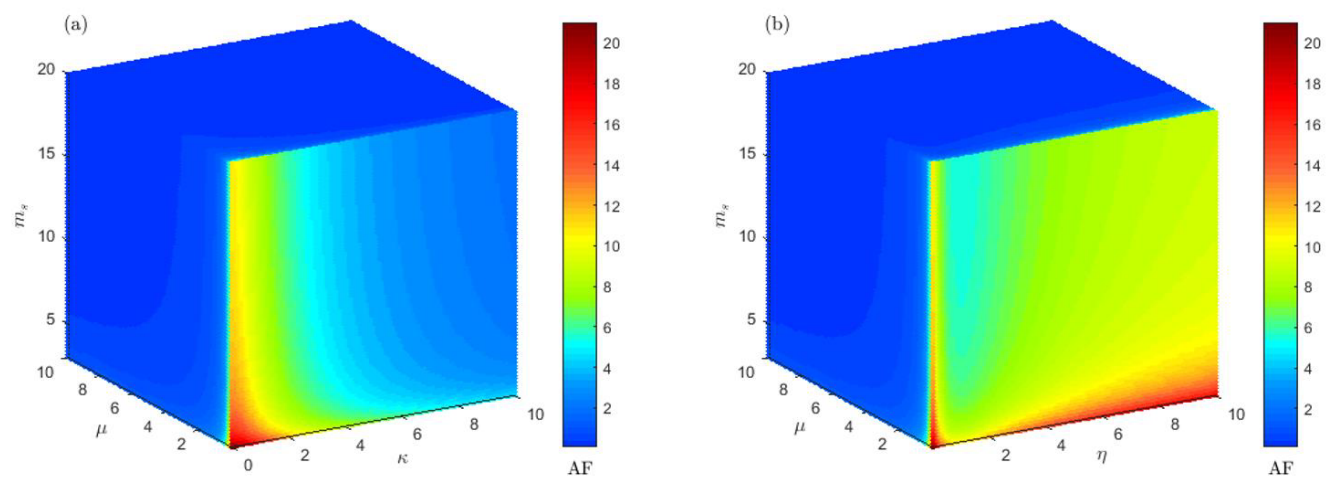

Fig. 3. AF in a (a) $\kappa-\mu /$ inverse gamma composite fading channel and an (b) $\eta-\mu /$ inverse gamma composite fading channel as a function of key parameters.
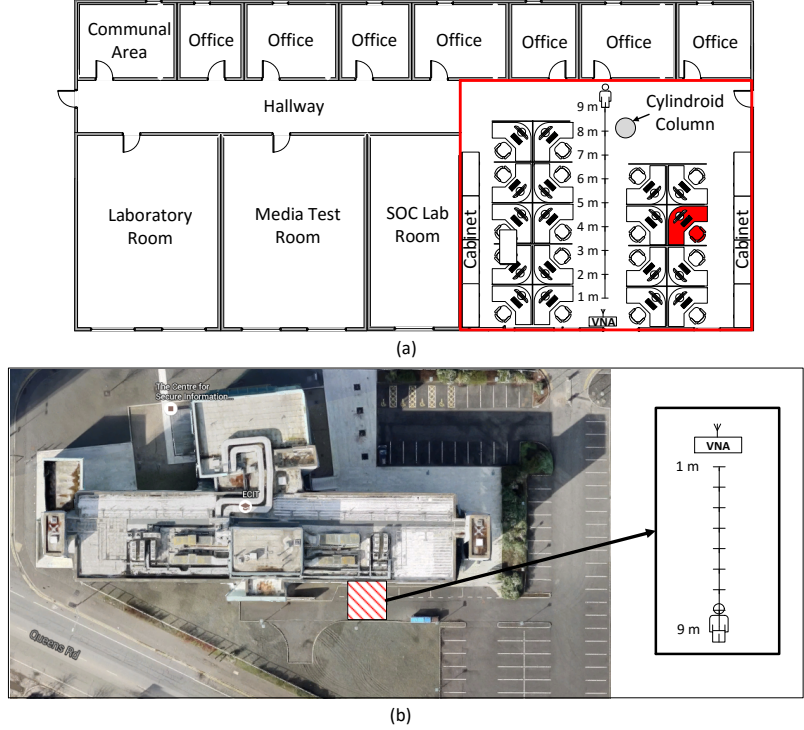

Fig. 4. (a) Indoor open office $\left(99.63 \mathrm{~m}^{2}\right)$ and (b) outdoor car parking environments used for the wearable off-body measurements. It should be noted that the desk filled with red color in (a) denotes that one person was working at his desk during the measurement in an open office area environment.

The first set of wearable off-body channel measurements were conducted in an indoor open office area environment [red rectangle: $10.62 \mathrm{~m} \times 12.23 \mathrm{~m}$, Fig. 4(a)] situated on the $1^{\text {st }}$ floor of the Institute of Electronics, Communications and Information Technology (ECIT) at Queen's University Belfast in the United Kingdom. The ECIT building consists of metal studded dry wall with a metal tiled floor covered with polypropylene fiber, rubber backed carpet tiles, and metal ceiling with mineral fiber tiles and recessed louvered luminaries suspended $2.70 \mathrm{~m}$ above floor level. As shown in Fig. 4(a), the open office area contained a number of soft partitions, cabinets, PCs, chairs and desks. During the channel measurements, one person was working at his desk. To improve the generality of the field validations conducted here, another set of measurements was performed in a more sparse environment, namely an outdoor car parking area adjacent to the ECIT building, as shown in Fig. 4(b).

The transmitter (TX) used for the measurements consisted of an ML5805 transceiver manufactured by RFMD, which was configured to transmit a continuous wave signal with an output power of $+17.6 \mathrm{dBm}$ at $5.8 \mathrm{GHz}$. During the wearable off- body measurements, the TX antenna was positioned on the front-central waist region of an adult male of height $1.83 \mathrm{~m}$ and mass $73 \mathrm{~kg}$ using a small strip of Velcro ${ }^{\circledR}$. For the receiver (RX), a single antenna was positioned on a nonconductive polyvinyl chloride (PVC) pole at height of 1.10 $\mathrm{m}$ above the floor level so that it was vertically polarized. It was then connected to port 1 of a Rohde \& Schwarz ZVB8 Vector Network Analyzer (VNA) using a low-loss coaxial cable. A pre-measurement calibration was conducted to reduce the effects of known system based errors using a Rohde \& Schwarz ZV-Z51 calibration unit. This also enabled the elimination of the effects of the power amplifier and cable loss. The VNA was configured as a sampling RX, recording the magnitude of the $b_{1}$ wave quantity incident on port 1 with a bandwidth of $10 \mathrm{kHz}$ (centered at the operation frequency of $5.8 \mathrm{GHz}$ ). The $b_{1}$ measurements were automatically collected and stored on a laptop through a local area network (LAN) connection, providing an effective channel sampling frequency of $425.6 \mathrm{~Hz}$. Both the TX and RX utilized identical omnidirectional sleeve dipole antennas with $+2.3 \mathrm{dBi}$ gain (Mobile Mark model PSKN3-24/55S). Two individual scenarios were considered for the LOS and NLOS channel conditions where the test subject walked towards and then away from the RX in a straight line (from the $9 \mathrm{~m}$ point to $1 \mathrm{~m}$ point and vice versa). It is worth remarking that the NLOS conditions corresponded to the condition where the human body obscured the direct communication path between the wearable node and the RX.

To abstract the composite fading signal for the wearable off-body measurements, the estimated path loss was removed from the raw measurement data using the log-distance path loss given in [36, Eq. (3.68)]. To this end, the elapsed time was first converted into a distance based upon an estimate of the test subject's velocity. The corresponding parameter estimates for the $\kappa-\mu /$ inverse gamma and $\eta-\mu /$ inverse gamma (Format 1) composite fading models were obtained using a non-linear least squares routine programmed in MATLAB to fit (3) and (12) to the wearable off-body measurement data. It should be noted that the minimum data set size used for the parameter estimations was 2331 for the wearable offbody channel measurements. The goodness-of-fit of these two models was evaluated using the RAD [37] which is a symmetric version of the Kullback-Leibler divergence (KLD) [38]. Unlike the KLD, the RAD satisfies the triangle inequality, which constitutes it a true distance metric. The RAD can be 
TABLE II

PARAmeter Estimates for the $\kappa-\mu$ / InVerse Gamma AND $\eta-\mu$ / InVERSE Gamma Composite Fading Models for All of the Wearable OfF-Body MEASUREMEnt Data ALONG With the RAD.

\begin{tabular}{|c|c|c|c|c|c|c|c|c|c|c|c|c|c|}
\hline \multirow{2}{*}{ Environment } & \multirow{2}{*}{$\begin{array}{l}\text { Channel } \\
\text { Conditions }\end{array}$} & \multicolumn{6}{|c|}{$\kappa-\mu /$ inverse gamma model } & \multicolumn{6}{|c|}{$\eta-\mu /$ inverse gamma model } \\
\hline & & $\kappa$ & $\mu$ & $m_{s}$ & $\Omega$ & RAD & $\sigma_{R}$ & $\eta$ & $\mu$ & $m_{s}$ & $\Omega$ & RAD & $\sigma_{R}$ \\
\hline \multirow{2}{*}{ Office } & LOS & 3.20 & 0.76 & 4.24 & 1.28 & 0.0012 & 1.05 & 1.00 & 0.70 & 9.71 & 1.46 & 0.0032 & 1.08 \\
\hline & NLOS & 1.50 & 0.77 & 2.40 & 1.49 & 0.0016 & 1.06 & 1.00 & 0.46 & 4.29 & 1.76 & 0.0020 & 1.07 \\
\hline \multirow{2}{*}{ Car Park } & LOS & 50.0 & 0.22 & 100.0 & 1.10 & 0.0025 & 1.07 & 1.00 & 2.81 & 100.0 & 1.13 & 0.0072 & 1.13 \\
\hline & NLOS & 0.70 & 0.93 & 2.91 & 1.50 & 0.0016 & 1.06 & 1.00 & 0.49 & 3.80 & 1.62 & 0.0014 & 1.05 \\
\hline
\end{tabular}
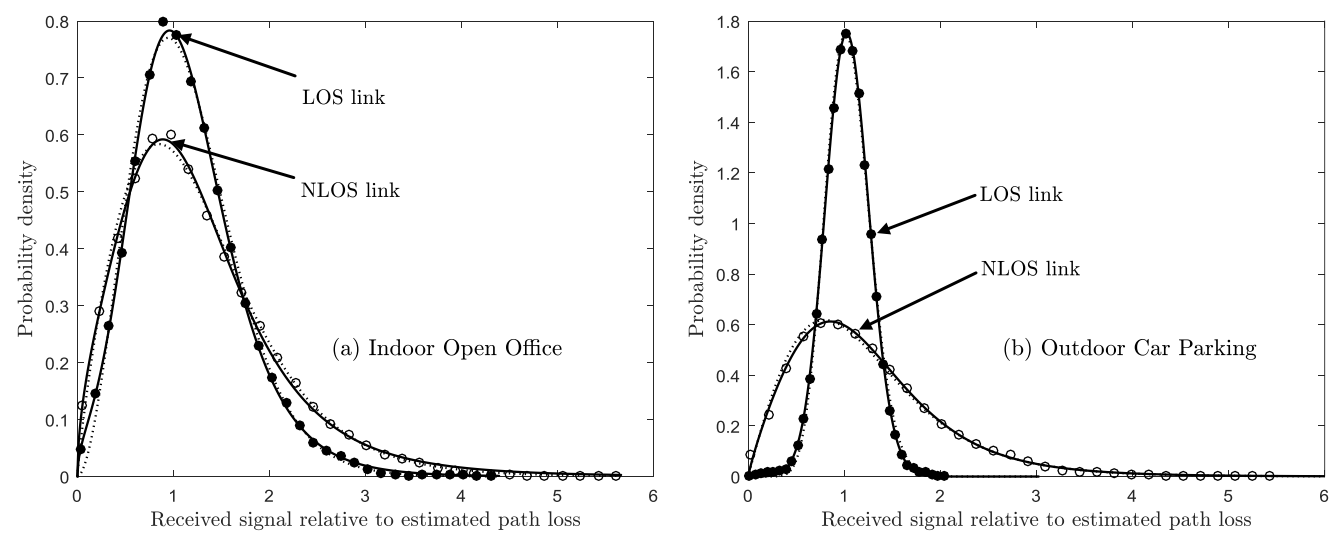

Fig. 5. Empirical PDFs (symbols) of the composite fading signal observed in the LOS and NLOS wearable off-body links for the (a) indoor open office and (b) outdoor car parking environments compared to the theoretical probability for the $\kappa-\mu /$ inverse gamma (continuous lines) and $\eta-\mu /$ inverse gamma (dotted lines) composite fading models.

defined as

$$
\operatorname{RAD}\left(f_{1}, f_{2}\right)=\left(\frac{1}{\operatorname{KLD}\left(f_{1}, f_{2}\right)}+\frac{1}{\operatorname{KLD}\left(f_{2}, f_{1}\right)}\right)^{-1}
$$

where $\operatorname{KLD}\left(f_{1}, f_{2}\right)=\int_{-\infty}^{\infty} f_{1}(x) \log _{2}\left(f_{1}(x) / f_{2}(x)\right) \mathrm{d} x$, with $f_{1}(x)$ and $f_{2}(x)$ denoting the true PDF of the measurement data and test PDF, i.e. the approximated PDF of $f_{1}(x)$, respectively. To assist with the interpretation of the goodnessof-fit, the equivalent RAD was used to calculate the standard deviation $\sigma_{R}$ of a zero-mean, $\sigma_{R}^{2}$ variance Gaussian PDF that is used to approximate a zero-mean, unit variance Gaussian PDF using the same approach used in [39].

As an example of the model fitting, Fig. 5 shows the PDFs of the $\kappa-\mu /$ inverse gamma and $\eta-\mu /$ inverse gamma composite fading models fitted to the measurement data for the LOS and NLOS wearable off-body channels within the two different environments. It is clear that both composite fading models provided a good fit to the empirical data for all of the considered cases. Referring to the RAD and $\sigma_{R}$ results presented in Table II, it has been shown that the $\kappa$ $\mu /$ inverse gamma composite model outperformed the $\eta-\mu$ / inverse gamma composite model for all of the wearable off-body measurements with the exception of the NLOS measurements in the outdoor car park. Table II also provides the parameter estimates for the $\kappa-\mu /$ inverse gamma and $\eta$ $\mu /$ inverse gamma models for all of the wearable off-body channels to allow the reader to reproduce their own simulated data based on the empirical data reported here. Remarkably, a resultant strong dominant signal component was found to exist for the NLOS wearable off-body channel in the indoor open office environment $(\kappa>1)$. However, there was no strong dominant signal component for the NLOS wearable off-body channel in the outdoor car parking environment $(\kappa<1)$. One possible explanation for this is that the indoor office environment contained a number of metal cabinets and was surrounded by metal reinforced ceiling and floor structures which can cause strong specular reflections, thereby creating dominant signal paths.

As shown in Table II, for both the indoor and outdoor environments, the $m_{s}$ parameters for the LOS links were greater than those for the NLOS links. Notably, the $m_{s}$ parameter estimate obtained for the indoor LOS link was much lower than that for the outdoor LOS link. This suggests that the indoor LOS radio link still experienced some shadowing effects even if a direct signal path existed. This observation was most likely caused by the fact that contributing signal components other than those arriving via LOS propagation, i.e. multipath components including both the dominant and scattered signal contributions, were shadowed by the human body and the surrounding obstacles in the indoor environment. On the contrary, the outdoor LOS link suffered less from a fluctuation of the mean signal power due to the relatively anechoic conditions which offered less opportunity for the generation of shadowed multipath components. For all of the considered wearable off-body channels, the scattered wave power of in-phase and quadrature components was found to be identical (i.e. $\eta=1$ ).

It is recalled that the $\kappa-\mu /$ gamma model has been utilized to characterize the composite fading behavior observed in wearable communications channels [20]. Motivated by this, for the LOS wearable off-body links, a cross-comparison between the $\kappa-\mu /$ inverse gamma and $\kappa-\mu /$ gamma models has been 
TABLE III

PARAMETER Estimates FOR THE $\kappa-\mu /$ GAMMA MOdel FOR THE LOS Wearable Off-Body Measurement Data ALONG With the RAD.

\begin{tabular}{|c|c|c|c|c|c|c|}
\hline \multirow{2}{*}{ Environmont } & \multicolumn{6}{|c|}{$\kappa-\mu /$ gamma model } \\
\cline { 2 - 7 } & $\kappa$ & $\mu$ & $a$ & $b$ & RAD & $\sigma_{R}$ \\
\hline Office & 4.04 & 0.65 & 3.97 & 0.40 & $\mathbf{0 . 0 0 1 3}$ & 1.05 \\
\hline Car Park & 50.0 & 0.22 & 100.0 & 0.01 & $\mathbf{0 . 0 0 2 5}$ & 1.07 \\
\hline
\end{tabular}

performed in terms of the goodness-of-fit. The corresponding parameter estimates ${ }^{3}$ for the $\kappa-\mu /$ gamma model are shown in Table III along with the RAD and $\sigma_{R}$ values. It is clear that there is no substantial difference between $\kappa, \mu$ and $m_{s}$ for the $\kappa-\mu /$ inverse gamma model and $\kappa, \mu$ and $a$ for the $\kappa-\mu$ / gamma model while there is a distinct difference between $\Omega$ and $b$ parameter. When comparing the RAD values between the $\kappa-\mu /$ inverse gamma and the $\kappa-\mu /$ gamma models, they were found to be almost the same, suggesting that the $\kappa$ $\mu$ / gamma model provided a comparable fit to the $\kappa-\mu$ / inverse gamma model. Nevertheless, the analytical forms of the fundamental statistics of the $\kappa-\mu /$ inverse gamma model are much more favorable as they are available in closed-form whereas those of the $\kappa-\mu /$ gamma model are not.

\section{Application II - Cellular Communications CHANNELS}

The use of device-to-device (D2D) communications has recently been proposed to supplement traditional cellular communications by providing higher data rates and extending the coverage of cellular networks [40]-[42]. In this context, D2D communications will be achieved by using network users themselves as ad hoc base stations to facilitate the routing of data traffic and to relay broadcasts. For the D2D measurements conducted in this study, the TX and RX antennas were securely fixed to the inside of a compact acrylonitrile butadiene styrene (ABS) enclosure $(107 \mathrm{~mm} \times 55 \mathrm{~mm} \times 20 \mathrm{~mm})$ using a small strip of Velcro ${ }^{\circledR}$. This configuration for the hypothetical User Equipment (UE) mimicked the form of a smart phone which allowed the user to emulate making a voice call as they would normally. Similar to the wearable off-body measurement set up, the wireless node used for the TX consisted of an ML5805 transceiver and was configured to transmit a continuous wave signal with an output power of $+17.6 \mathrm{dBm}$ at $5.8 \mathrm{GHz}$. The wireless node used for the RX also featured an ML5805 transceiver attached to a PIC32MX which acted as a baseband controller, allowing the analog received signal strength (RSS) output to be sampled with a 10-bit quantization depth at a rate of $10 \mathrm{kHz}$. The utilized TX and RX antennas were the same as those used for the wearable off-body channel measurements which were connected to the wireless nodes using low-loss coaxial cables.

The D2D measurements were performed at $5.8 \mathrm{GHz}$ in two different environments, as shown in Fig. 6, namely (a) an indoor open office area environment and (b) an outdoor

\footnotetext{
${ }^{3}$ It is worth highlighting that all parameters for the $\kappa-\mu /$ gamma model $(\kappa, \mu, a, b)$ were also estimated using a non-linear least squares routine programmed in MATLAB where the integral contained in the $\kappa-\mu /$ gamma model was computed using the trapz function which is also available in MATLAB.
}

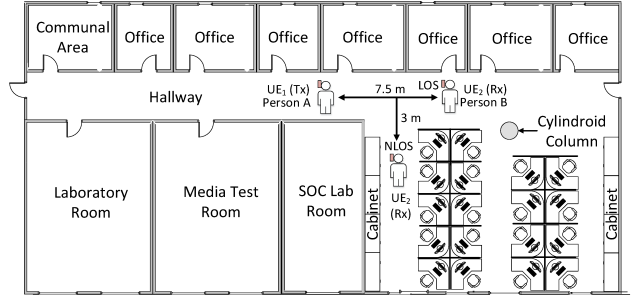

(a)

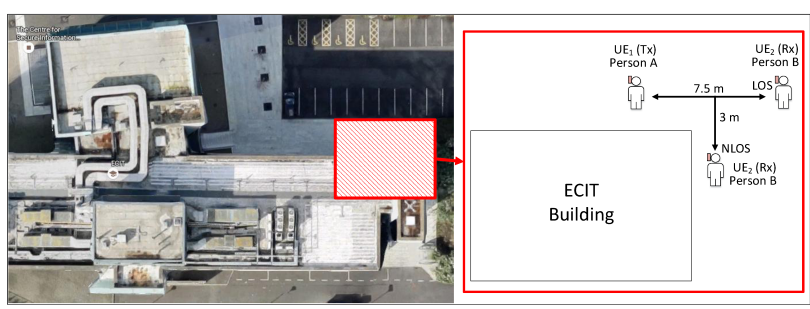

(b)

Fig. 6. D2D measurements in (a) an indoor open office environment and (b) an outdoor open space environment showing different locations of person B $\left(\mathrm{UE}_{2}\right)$ for the LOS and NLOS cases.

open space environment. The open office area is the same environment where the wearable off-body measurements were conducted. During the D2D measurements, the open office area was unoccupied in order to facilitate pedestrian free D2D channel measurements. The outdoor D2D measurements were conducted in an open space close to the ECIT building. In this study, the D2D link was formed between two persons, namely person $\mathrm{A}$, an adult male of height $1.83 \mathrm{~m}$ and weight $73 \mathrm{~kg}$, and person $\mathrm{B}$, a female of height $1.65 \mathrm{~m}$ and weight $51 \mathrm{~kg}$. It should be noted that the UEs used by persons A and B are denoted as $\mathrm{UE}_{1}$ and $\mathrm{UE}_{2}$, respectively. For all of the D2D measurements, persons A and B held the respective UE at their left ear to imitate making a voice call. Both test subjects were initially stationary, after which they were instructed to walk around randomly within a circle of radius of $0.5 \mathrm{~m}$ from their starting points. It is worth highlighting that for the LOS D2D measurements in both environments, while there may have been a direct LOS between the two person's bodies during the trials, in actual fact, the link between the hypothetical UEs would have been subject to quasi-LOS conditions due to the random movements undertaken. For the NLOS case, person B was always positioned around an adjacent corner to ensure that the NLOS conditions (i.e. no direct path between persons A and B) were maintained irrespective of the random movements.

In the analysis of the D2D channels, the global mean signal power was removed from the D2D measurement data to abstract the composite fading signal for field measurement data. Again, all parameter estimates for the $\kappa-\mu /$ inverse gamma and $\eta-\mu /$ inverse gamma models were obtained using a non-linear least squares routine programmed in MATLAB to fit (3) and (12) to the D2D measurement data. It should be noted that the minimum data set size used for the parameter estimations was 138148 for the D2D measurements. The corresponding RAD and $\sigma_{R}$ were again utilized to evaluate the goodness-of-fit of these two models with the D2D measurement data.

As an example of the model fitting process, Figs. 7(a) 
TABLE IV

Parameter Estimates for the $\kappa-\mu$ / Inverse Gamma And $\eta-\mu$ / InVERSe Gamma Composite Fading Models for All of the D2D MEASUREMENT Data ALONG With THE RAD.

\begin{tabular}{|c|c|c|c|c|c|c|c|c|c|c|c|c|c|}
\hline \multirow{2}{*}{ Environments } & \multirow{2}{*}{$\begin{array}{c}\text { Channel } \\
\text { Conditions }\end{array}$} & \multicolumn{6}{|c|}{$\kappa-\mu$ / inverse gamma model } & \multicolumn{6}{|c|}{$\eta-\mu /$ inverse gamma model } \\
\hline & & $\kappa$ & $\mu$ & $m_{s}$ & $\Omega$ & RAD & $\sigma_{R}$ & $\eta$ & $\mu$ & $m_{s}$ & $\Omega$ & RAD & $\sigma_{R}$ \\
\hline \multirow{2}{*}{ Indoor } & LOS & 1.76 & 0.86 & 1.21 & 0.62 & 0.0049 & 1.10 & 1.00 & 0.56 & 1.42 & 0.70 & 0.0054 & 1.11 \\
\hline & NLOS & 0.01 & 0.76 & 4.14 & 1.19 & 0.0045 & 1.10 & 4.48 & 0.39 & 50.0 & 1.50 & 0.0040 & 1.09 \\
\hline \multirow{2}{*}{ utdoor } & LOS & 2.48 & 0.76 & 1.14 & 0.64 & 0.0122 & 1.17 & 1.00 & 0.58 & 1.33 & 0.73 & 0.0144 & 1.19 \\
\hline & NLOS & 0.01 & 0.95 & 1.11 & 0.55 & 0.0048 & 1.10 & 4.73 & 0.51 & 1.30 & 0.64 & 0.0047 & 1.10 \\
\hline
\end{tabular}
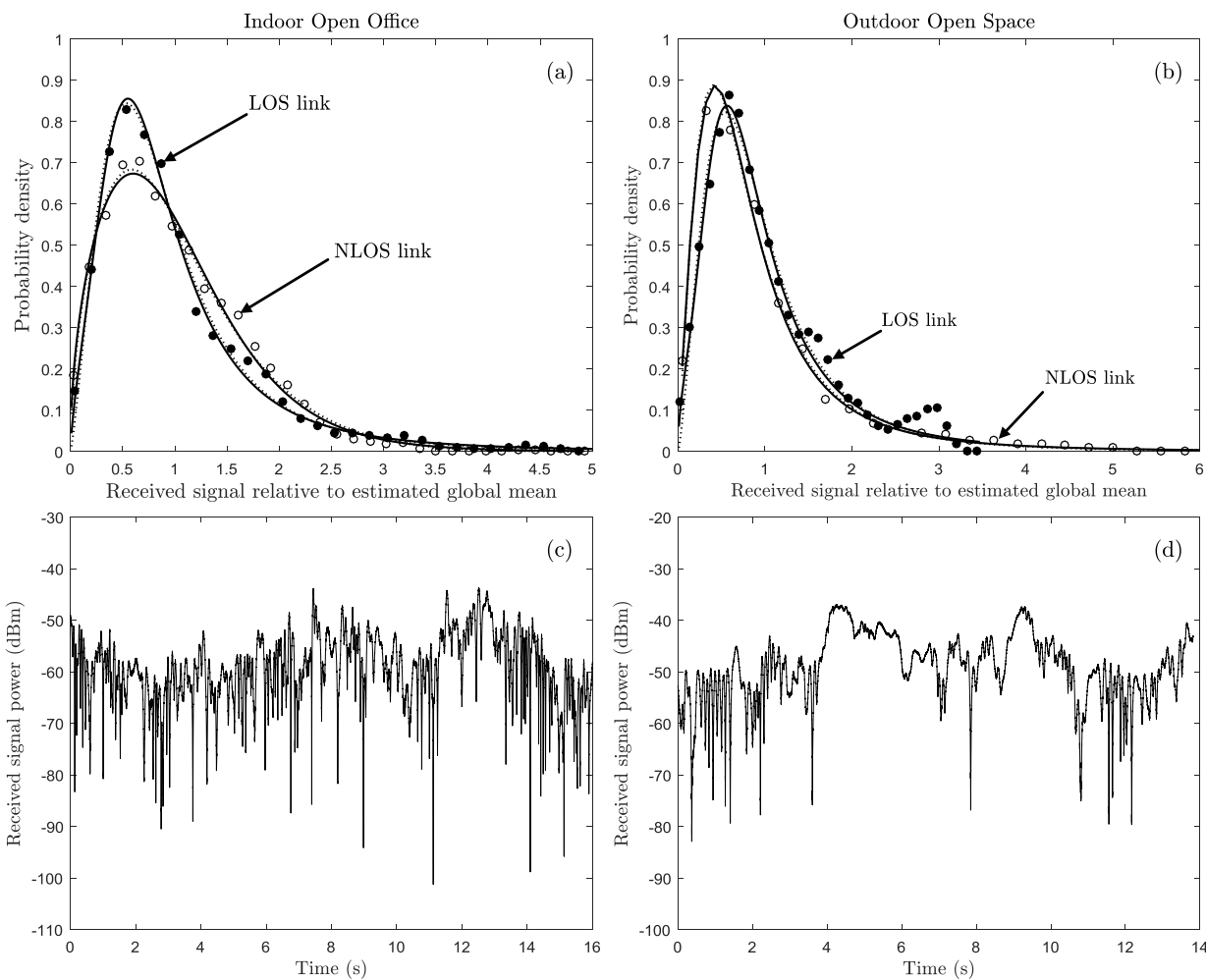

Fig. 7. Empirical (symbols) and theoretical PDFs for the $\kappa-\mu /$ inverse gamma (continuous lines) and $\eta-\mu$ / inverse gamma (dotted lines) models for the LOS and NLOS D2D links in the (a) indoor open office and (b) outdoor open space environments alongside the received signal power for the LOS link in the (c) indoor open office and (d) outdoor open space environments.

and (b) show the PDFs of the $\kappa-\mu /$ inverse gamma and $\eta-\mu /$ inverse gamma composite fading models fitted to the measurement data for both the LOS and NLOS D2D channels in the indoor and outdoor environments, respectively. It can be seen that both models provided an adequate fit to the measurement data for both the LOS and NLOS conditions. Table IV shows the parameter estimates for the $\kappa-\mu /$ inverse gamma and $\eta-\mu /$ inverse gamma models for all of the D2D channels along with their RADs and $\sigma_{R}$. For both the indoor and outdoor environments, the $m_{s}$ parameters for the LOS cases were observed to be quite low, indicating that the LOS link was subject to considerable shadowing. This is understandable due to the constantly changing orientation and posture of both persons and it can also be observed in the measured received signal power time series for the LOS cases in both the indoor and outdoor environments presented in Figs. 7(c) and (d). From these plots, it is clear that the received signal experienced longer-term fading due to the shadowing caused by both users, strongly advocating the use of a composite fading model in these cases.
The $\kappa$ parameter estimates indicate that there existed strong dominant signal components for both the indoor and outdoor LOS links $(\kappa>1)$, but not for the NLOS links $(\kappa<1)$. When considering the $\eta$ parameter, the scattered wave power of in-phase and quadrature components was identical for both the indoor and outdoor LOS links $(\eta=1)$, but not identical for the indoor and outdoor NLOS links $(\eta \neq 1)$. Although both composite fading models visually provided an adequate fit to the measured data, interpreting the RAD and $\sigma_{R}$ results presented in Table IV, it can be seen that the $\kappa-\mu /$ inverse gamma model provided a better fit to the LOS measurement data, whereas the $\eta-\mu$ / inverse gamma model provided a better fit to the NLOS measurement data.

\section{Application III - Vehicular Communications CHANNELS}

Vehicular communications have become increasingly popular due to their potential for improving traffic safety and avoiding congestion [43], [44]. In this part of the study, vehicle-to-vehicle (V2V) communications channels, which are 

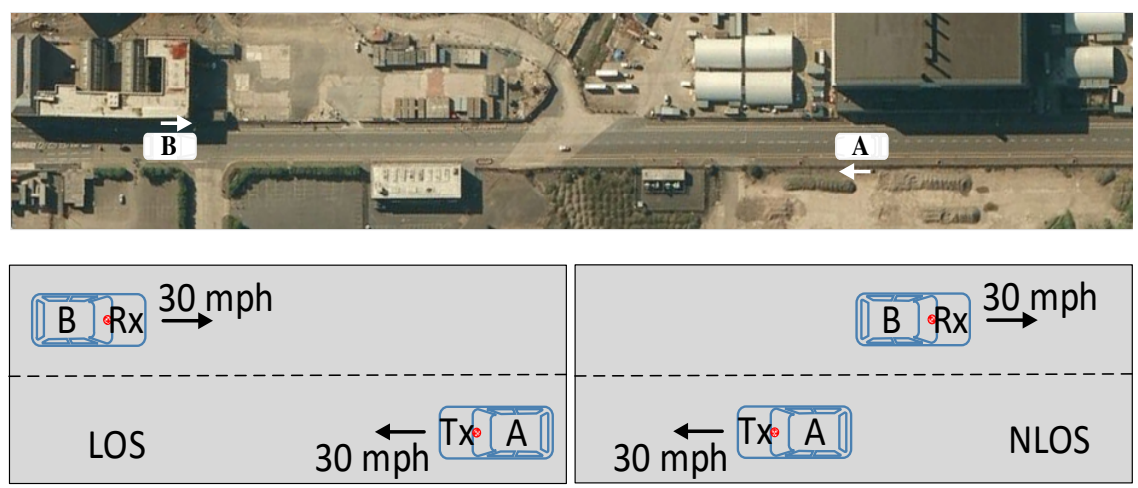

Fig. 8. Satellite view of $\mathrm{V} 2 \mathrm{~V}$ measurement environment showing the position of two vehicles and two different scenarios (LOS and NLOS channel conditions). It should be noted that the road is approximately $10 \mathrm{~m}$ in width.

key components of vehicular networks, were considered. V2V channels exist between wireless devices situated on one vehicle and those situated on another vehicle. For the V2V channel measurements, the utilized TX was the same as that considered in Sections III and IV which was configured to generate a continuous wave signal with an output power of $+17.6 \mathrm{dBm}$ at $5.8 \mathrm{GHz}$. The RX was the identical wireless node used for the D2D measurements, but unlike the D2D measurements, the channel sampling frequency was $1 \mathrm{kHz}$. The V2V measurements were conducted in a business district environment in the Titanic Quarter of Belfast in the United Kingdom as shown in Fig. 8. For the V2V measurements, the TX was positioned on the center of the dash board of vehicle A, namely a Vauxhall (Opel in continental Europe) Zafira SRi using a small strip of Velcro ${ }^{\circledR}$ while the RX was mounted on the dash board of vehicle B, namely a Vauxhall Astra SRi. The measurement area consisted of a straight road with a number of office buildings nearby. To create the LOS and $\mathrm{NLOS}^{4}$ channel conditions, both vehicles A and B initially moved towards each other with a speed of $30 \mathrm{mph}$ before passing and continuing their onward journey as shown in Fig. 8. A distance of $50 \mathrm{~m}$ in either side of the intersection point was considered for the LOS and NLOS analysis performed in this study. Although the V2V channel measurements were performed during off-peak traffic hours, there still existed pedestrians and other vehicular traffic in the vicinity of vehicles A and B.

Similar to the wearable off-body analysis, the estimated path loss was removed from the $\mathrm{V} 2 \mathrm{~V}$ measurement data using the log-distance path loss to abstract the composite fading signal from the field measurement data. To this end, the elapsed time was first converted into a distance based upon the vehicle's velocity. As before, the parameter estimates for the requisite models were obtained using the procedure outlined in Sections III and IV. It should be noted that the minimum data set size used for the parameter estimations was 13287 for the V2V measurements. As an example of the model fitting process, Fig. 9 shows the PDFs of the $\kappa-\mu /$ inverse gamma and $\eta-\mu$ / inverse gamma composite fading models fitted to the LOS

\footnotetext{
${ }^{4}$ Although we categorize the link between the two vehicles as NLOS for the case when vehicles A and B moved away from each other, we make the point that the link between the two vehicles could have been subject to quasi-LOS conditions due to the positioning of the TX and RX which were equipped with omnidirectional sleeve dipole antennas.
}

and NLOS V2V measurement data in conjunction with their respective received signal power time series.

Also shown is the estimated path loss which was calculated using a reference distance of $5 \mathrm{~m}$, which was the separation distance between the TX on vehicle $\mathrm{A}$ and the RX on vehicle B. It can be seen that both the $\kappa-\mu /$ inverse gamma and $\eta-\mu /$ inverse gamma composite fading models were in good agreement with the LOS and NLOS measurement data. Table $\mathrm{V}$ provides the associated parameter estimates for both models over all of the $\mathrm{V} 2 \mathrm{~V}$ channels along with the computed RAD and $\sigma_{R}$. For both the LOS and NLOS links, strong dominant signal components were found to exist $(\kappa>1)$ and the scattered wave power of the in-phase and quadrature components was observed to be identical $(\eta=1)$. Consulting the RAD and $\sigma_{R}$ results, it can be seen that the $\kappa-\mu /$ inverse gamma model provided a better fit to both the LOS and NLOS measurement data than the $\eta-\mu /$ inverse gamma model.

\section{CONCLUSION}

Two composite fading models, namely $\kappa-\mu$ / inverse gamma and $\eta-\mu /$ inverse gamma distributions, have been presented. The $\kappa-\mu /$ inverse gamma model assumes that the mean power of both the dominant and scattered signal components is subject to shadowing which is weighted by an inverse gamma RV. On the other hand, the $\eta-\mu$ / inverse gamma model assumes that the mean power of the scattered component is subject to shadowing which is also weighted by an inverse gamma RV. Both composite fading models include well-known distributions as special cases when they coincide with the $\kappa-\mu$ and $\eta-\mu$ fading models, respectively. Most importantly though, it has been shown that they are also able to approximate other composite fading models commonly encountered in the literature.

Novel analytic expressions have been derived for the associated statistical measures of interest while the utility of the proposed models has been validated using a diverse range of field measurements for emerging wireless applications such as wearable, cellular D2D and V2V communications. In particular, we have considered the composite fading signal observed in LOS and NLOS channels for these use cases. By considering the composite fading signal, we were able to take into account the simultaneous impact of multipath and 
TABLE V

Parameter Estimates for the $\kappa-\mu$ / InVerse Gamma AND $\eta-\mu$ / InVERSE Gamma Composite Fading Models for All OF the V2V MEASUREMENT Data ALONG With THE RAD.

\begin{tabular}{|c|c|c|c|c|c|c|c|c|c|c|c|c|}
\hline \multirow{2}{*}{ Channel Conditions } & \multicolumn{4}{|c|}{$\kappa-\mu /$ inverse gamma model } & \multicolumn{4}{c|}{$\eta$ - $/$ inverse gamma model } \\
\cline { 2 - 14 } & $\kappa$ & $\mu$ & $m_{s}$ & $\Omega$ & RAD & $\sigma_{R}$ & $\eta$ & $\mu$ & $m_{s}$ & $\Omega$ & RAD & $\sigma_{R}$ \\
\hline LOS & 1.02 & 0.74 & 100.00 & 2.04 & $\mathbf{0 . 0 0 2 4}$ & 1.07 & 1.00 & 0.43 & 100.00 & 2.18 & 0.0096 & 1.15 \\
\hline \hline NLOS & 1.02 & 0.83 & 1.63 & 1.32 & $\mathbf{0 . 0 0 4 4}$ & 1.10 & 1.00 & 0.45 & 2.01 & 1.48 & 0.0050 & 1.11 \\
\hline
\end{tabular}
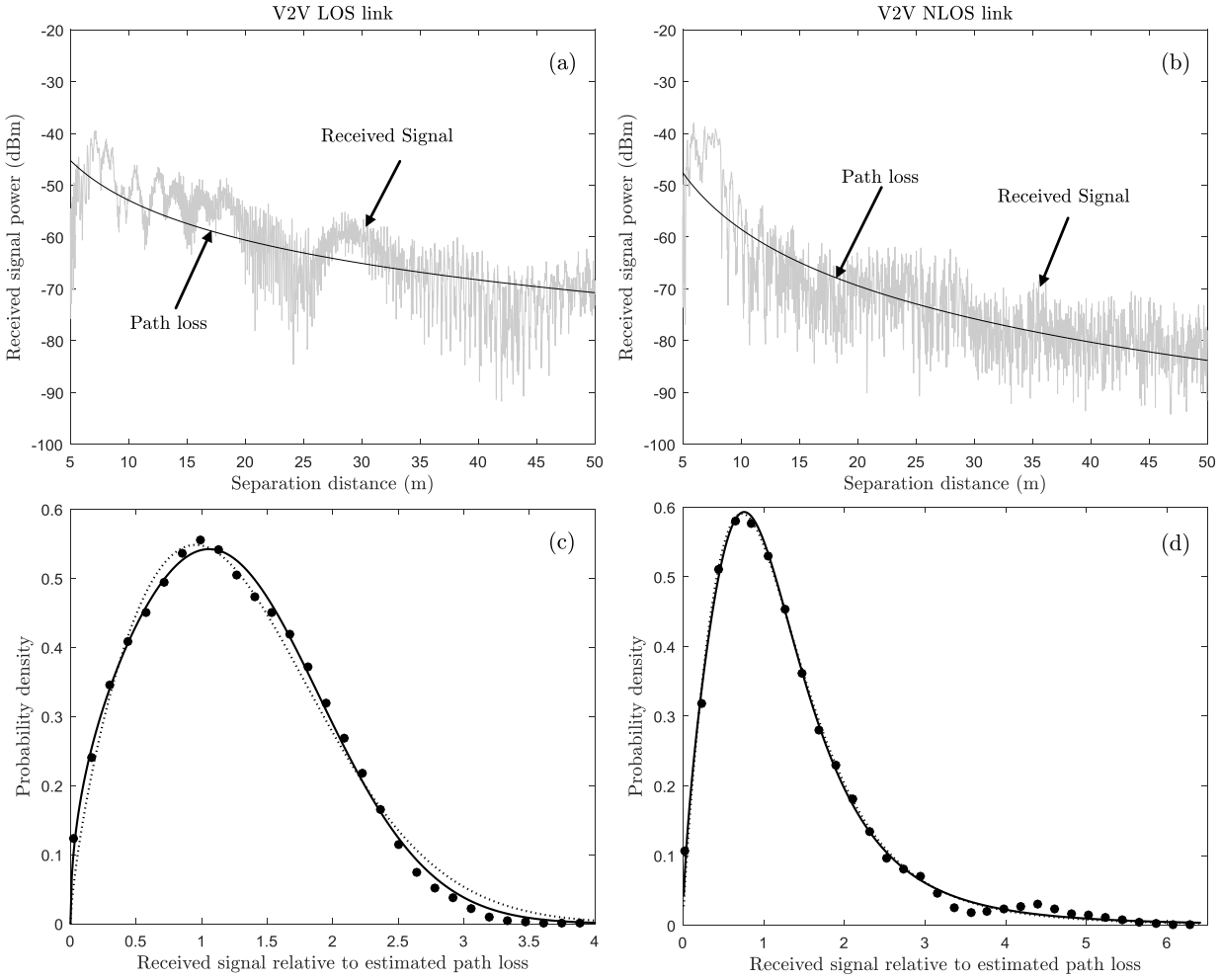

Fig. 9. Received signal power with a superimposed path loss fit for (a) the V2V LOS link and (b) the V2V NLOS link and empirical (circles) and theoretical PDFs for the $\kappa-\mu$ / inverse gamma (continuous lines) and $\eta-\mu$ / inverse gamma (dotted lines) models for (c) the V2V LOS link and (d) the V2V NLOS link.

shadowing. Over all of the field measurements undertaken, the PDFs of the $\kappa-\mu$ / inverse gamma and the $\eta-\mu /$ inverse gamma models have been shown to provide an impressive fit to the composite fading, thereby providing a physical validation for these new models.

Using the RAD and $\sigma_{R}$, it has been shown that the $\kappa$ $\mu /$ inverse gamma model provided a better fit compared to the $\eta-\mu$ / inverse gamma model when strong dominant signal components existed $(\kappa>1)$ and the scattered wave power of the in-phase and quadrature components of each cluster of multipath were identical $(\eta=1)$. In contrast, the $\eta-\mu /$ inverse gamma model outperformed the $\kappa-\mu /$ inverse gamma model when there were no resultant strong dominant signal components $(\kappa<1)$ and/or the scattered wave power of the in-phase and quadrature components of each multipath cluster were not identical $(\eta \neq 1)$. Nonetheless, there were a couple of instances where the $\kappa-\mu /$ inverse gamma model provided a marginally better fit than the $\eta-\mu /$ inverse gamma model for the NLOS conditions (i.e. the indoor wearable and V2V NLOS channels), however it is worth remarking that in these cases the difference in the fit provided by the two models is virtually indistinguishable. Overall, these results suggest that the key factor in determining which model provides a better fit to the measured data is the presence of strong dominant signal components and/or the difference in the scattered wave power of the in-phase and quadrature components of each multipath cluster, rather than the presence of a direct signal path.

\section{APPENDIX A \\ Proofs of Fundamental STATISTICS OF THE $\kappa-\mu /$ INVERSE GAMMA MODEL}

\section{A. Proof of Theorem 1}

The PDF of the composite signal envelope in a $\kappa-\mu$ / inverse gamma channel, $R$, can be determined by averaging the infinite integral of the conditional probability density of the $\kappa-\mu$ fading process with respect to the random variation of the mean signal power, $A$, as follows

$$
f_{R}(r)=\int_{0}^{\infty} f_{R \mid A}(r \mid \alpha) f_{A}(\alpha) \mathrm{d} \alpha
$$

where, using the signal model given in (1), this insinuates that

$$
\begin{aligned}
f_{R \mid A}(r \mid \alpha)= & \frac{2 \mu(\kappa+1)^{\frac{\mu+1}{2}} r^{\mu}}{\kappa^{\frac{\mu-1}{2}} \exp (\mu \kappa)(\alpha \Omega)^{\frac{\mu+1}{2}}} \exp \left(-\mu(\kappa+1) \frac{r^{2}}{\alpha \Omega}\right) \\
& \times I_{\mu-1}\left(2 \mu \sqrt{\kappa(\kappa+1)} \frac{r}{\sqrt{\alpha \Omega}}\right)
\end{aligned}
$$


where $I_{v}(\cdot)$ denotes the modified Bessel function of the first kind and order $v$ [45, Eq. (9.6.20)]. Substituting (29) and (2) in (28), the PDF of the $\kappa-\mu /$ inverse gamma model can be written as

$$
\begin{aligned}
f_{R}(r)= & \frac{2 \mu(\kappa+1)^{\frac{\mu+1}{2}} m_{s}{ }^{m_{s}} r^{\mu}}{\kappa^{\frac{\mu-1}{2}} \exp (\mu \kappa) \Gamma\left(m_{s}\right) \Omega^{\frac{\mu+1}{2}}} \int_{0}^{\infty}\left(\frac{1}{\alpha}\right)^{\frac{2 m_{s}+\mu+3}{2}} \\
& \times \exp \left(-\mu(\kappa+1) \frac{r^{2}}{\alpha \Omega}\right) \exp \left(-\frac{m_{s}}{\alpha}\right) \\
& \times I_{\mu-1}\left(2 \mu \sqrt{\kappa(\kappa+1)} \frac{r}{\sqrt{\alpha \Omega}}\right) \mathrm{d} \alpha .
\end{aligned}
$$

Performing a simple transformation of variables and applying [46, Eq. (2.15.5.4)] along with some algebraic manipulation, (30) can be rewritten in closed-form as given in (3).

\section{B. Proof of Theorem 2}

By expanding the Kummer confluent hypergeometric function in (4) in terms of the series representation [31, Eq. (07.20.02.0001.01)], the CDF of the instantaneous SNR of the $\kappa-\mu /$ inverse gamma model can be expressed as

$$
\begin{aligned}
F_{\gamma}(\gamma) & =\int_{0}^{\gamma} \frac{\exp (-\mu \kappa) \mu^{\mu}(\kappa+1)^{\mu}\left(m_{s} \bar{\gamma}\right)^{m_{s}} \gamma^{\mu-1}}{B\left(m_{s}, \mu\right)\left[\mu(\kappa+1) \gamma+m_{s} \bar{\gamma}\right]_{s}^{m_{s}+\mu}} \\
& \times \sum_{i=0}^{\infty} \frac{\left(m_{s}+\mu\right)_{i}}{i !(\mu)_{i}}\left(\frac{\mu^{2} \kappa(\kappa+1) \gamma}{\mu(\kappa+1) \gamma+m_{s} \bar{\gamma}}\right)^{i} \mathrm{~d} \gamma .
\end{aligned}
$$

With the aid of [26, Eq. (3.194.5)], we can obtain the CDF of the instantaneous SNR of the $\kappa-\mu /$ inverse gamma composite fading model as given in (5). By expanding the Gauss hypergeometric function in (5) in terms of an infinite series representation [31, Eq. (07.23.02.0001.01)], ${ }_{2} F_{1}(a, b ; c ; z)=$ $\sum_{i=0}^{\infty} \frac{(a)_{i}(b)_{i} z^{i}}{(c)_{i} i !}$ for $|z|<1$, and using the definition of the Kampé de Fériet function [29], the CDF of the instantaneous SNR of the $\kappa-\mu /$ inverse gamma model can be obtained in closed-form as given in (6). On the contrary, for $|z| \geq 1$, the Gauss hypergeometric function in (5) is expanded as follows [31, Eq. (07.23.02.0004.01)],

$$
\begin{aligned}
{ }_{2} F_{1}(a, b ; c ; x) & =\frac{\Gamma(b-a) \Gamma(c)}{\Gamma(b) \Gamma(c-a)} \sum_{l=0}^{\infty} \frac{(a)_{l}(a-c+1)_{l} x^{-l}}{l !(a-b+1)_{l}(-x)^{a}} \\
& +\frac{\Gamma(a-b) \Gamma(c)}{\Gamma(a) \Gamma(c-b)} \sum_{l=0}^{\infty} \frac{(b)_{l}(b-c+1)_{l} x^{-l}}{l !(b-a+1)_{l}(-x)^{b}} .
\end{aligned}
$$

Consequently, using (32) and the definition of the Humbert $\Psi_{1}$ function [30], the CDF of the instantaneous SNR of the $\kappa-\mu /$ inverse gamma model can be obtained as given in (7).

\section{Proof of Theorem 3}

In a similar manner to Appendix A-B, the higher order moments of the instantaneous SNR of the $\kappa-\mu /$ inverse gamma model can be expressed using an exact infinite series expansion of the Kummer confluent hypergeometric function [31, Eq. $(07.20 .02 .0001 .01)]$ such that

$$
\begin{aligned}
\mathbb{E}\left[\gamma^{n}\right] & =\int_{0}^{\infty} \frac{\exp (-\mu \kappa) \mu^{\mu}(\kappa+1)^{\mu}\left(m_{s} \bar{\gamma}\right)^{m_{s}} \gamma^{\mu+n-1}}{B\left(m_{s}, \mu\right)\left[\mu(\kappa+1) \gamma+m_{s} \bar{\gamma}\right]_{s}^{m_{s}+\mu}} \\
& \times \sum_{i=0}^{\infty} \frac{\left(m_{s}+\mu\right)_{i}}{i !(\mu)_{i}}\left(\frac{\mu^{2} \kappa(\kappa+1) \gamma}{\mu(\kappa+1) \gamma+m_{s} \bar{\gamma}}\right)^{i} \mathrm{~d} \gamma .
\end{aligned}
$$

Using [26, Eq. (3.251.11)] and the series representation of the Kummer confluent hypergeometric function [31, Eq. (07.20.02.0001.01)], we obtain the higher order moments of the instantaneous SNR of the $\kappa-\mu /$ inverse gamma model in closed-form expression as given in (8).

\section{Proof of Corollary 1}

The corresponding AF can be obtained using the definition [1, Eq. (1.27)], $\mathrm{AF}=\frac{\mathbb{E}\left[\gamma^{2}\right]}{\left.\mathbb{E}^{2} \gamma\right]}-1$, where $\mathbb{E}[\gamma]$ and $\mathbb{E}\left[\gamma^{2}\right]$ denote the first and second moments, respectively. By substituting $n=1$ and $n=2$ in (8) we obtain

$$
\mathbb{E}[\gamma]=\left(\frac{m_{s} \bar{\gamma}}{\mu(\kappa+1)}\right) \frac{B\left(m_{s}-1, \mu+1\right)}{\exp (\mu \kappa) B\left(m_{s}, \mu\right)}{ }_{1} F_{1}(\mu+1 ; \mu ; \mu \kappa)
$$

and

$$
\mathbb{E}\left[\gamma^{2}\right]=\left(\frac{m_{s} \bar{\gamma}}{\mu(\kappa+1)}\right)^{2} \frac{B\left(m_{s}-2, \mu+2\right)}{\exp (\mu \kappa) B\left(m_{s}, \mu\right)}{ }_{1} F_{1}(\mu+2 ; \mu ; \mu \kappa) .
$$

Using Kummer's transformation [31, Eq. (07.20.17.0013.01)], (34) and (35) can be rewritten as

$$
\mathbb{E}[\gamma]=\left(\frac{m_{s} \bar{\gamma}}{\mu(\kappa+1)}\right) \frac{B\left(m_{s}-1, \mu+1\right)}{B\left(m_{s}, \mu\right)}{ }_{1} F_{1}(-1 ; \mu ;-\mu \kappa)
$$

and

$$
\mathbb{E}\left[\gamma^{2}\right]=\left(\frac{m_{s} \bar{\gamma}}{\mu(\kappa+1)}\right)^{2} \frac{B\left(m_{s}-2, \mu+2\right)}{B\left(m_{s}, \mu\right)}{ }_{1} F_{1}(-2 ; \mu ;-\mu \kappa) .
$$

Now, using the special representation of the Kummer hypergeometric function, ${ }_{1} F_{1}(-1 ; b ; z)=1-\frac{z}{b}$ [31, Eq. (07.20.03.0018.01)] and ${ }_{1} F_{1}(-2 ; b ; z)=1-\frac{2 z}{b}+\frac{z^{2}}{b(1+b)}$ [31, Eq. (07.20.03.0017.01)], we obtain the AF of the $\kappa-\mu /$ inverse gamma model in closed-form as given in (9).

\section{E. Proof of Theorem 4}

The MGF can be derived from (4) using an infinite series expansion of the Kummer confluent hypergeometric function [31, Eq. (07.20.02.0001.01)] yielding

$$
\begin{aligned}
& M_{\gamma}(-s)=\sum_{i=0}^{\infty} \frac{\left(m_{s}+\mu\right)_{i}\left[\mu^{2} \kappa(\kappa+1) \gamma\right]^{i}}{i !(\mu)_{i}\left[\mu(\kappa+1) \gamma+m_{s} \bar{\gamma}\right]^{i}} \\
& \times \int_{0}^{\infty} \frac{\exp (-\mu \kappa-s \gamma) \mu^{\mu}(\kappa+1)^{\mu}\left(m_{s} \bar{\gamma}\right)^{m_{s}} \gamma^{\mu-1}}{B\left(m_{s}, \mu\right)\left[\mu(\kappa+1) \gamma+m_{s} \bar{\gamma}\right]^{m_{s}+\mu}} \mathrm{d} \gamma .
\end{aligned}
$$

With the aid of [26, Eq. (3.383.5)] and making use of the generalized Laguerre polynomial [31, Eq. (07.03.02.0001.01)], we can obtain the corresponding MGF of the $\kappa-\mu /$ inverse gamma composite fading model as follows

$$
\begin{aligned}
M_{\gamma}(-s)=\sum_{i=0}^{\infty} & \frac{(\mu \kappa)^{i}}{i ! \exp (\mu \kappa)}\left[{ }_{1} F_{1}\left(\mu+i ; 1-m_{s} ; \frac{s m_{s} \bar{\gamma}}{\mu(\kappa+1)}\right)\right. \\
+ & \left(\frac{s m_{s} \bar{\gamma}}{\mu(\kappa+1)}\right)^{m_{s}} \frac{\Gamma\left(-m_{s}\right)}{B\left(m_{s}, \mu+i\right)} \\
& \left.\times{ }_{1} F_{1}\left(m_{s}+\mu+i ; 1+m_{s} ; \frac{s m_{s} \bar{\gamma}}{\mu(\kappa+1)}\right)\right] .
\end{aligned}
$$

By using the series form of the Kummer confluent hypergeometric function [31, Eq. (07.20.02.0001.01)] in (39) and the definition of the Humbert $\Psi_{2}$ function [30], the MGF of the 
$\kappa-\mu /$ inverse gamma model can be expressed in closed-form as given in (10).

\section{APPENDIX B}

PROOFS OF Fundamental Statistics of THE $\eta$ - $\mu$ / INVERSE GAMMA MODEL

\section{A. Proof of Theorem 5}

Using the signal model given in (11), for the $\eta-\mu /$ inverse gamma model, this insinuates that

$$
\begin{aligned}
f_{R \mid A}(r \mid \alpha)= & \frac{4 \sqrt{\pi} \mu^{\mu+\frac{1}{2}} h^{\mu} r^{2 \mu}}{\Gamma(\mu) H^{\mu-\frac{1}{2}}(\alpha \Omega)^{\mu+\frac{1}{2}}} \\
& \times \exp \left(-\frac{2 \mu h r^{2}}{\alpha \Omega}\right) I_{\mu-\frac{1}{2}}\left(\frac{2 \mu H r^{2}}{\alpha \Omega}\right) .
\end{aligned}
$$

By substituting (40) and (2) into (28), the PDF of the composite fading signal in an $\eta-\mu /$ inverse gamma composite fading channel, $R$, can be expressed as follows

$$
\begin{aligned}
f_{R}(r)= & \frac{4 \sqrt{\pi} \mu^{\mu+\frac{1}{2}} h^{\mu} m_{s} m_{s} r^{2 \mu}}{\Gamma(\mu) \Gamma\left(m_{s}\right) H^{\mu-\frac{1}{2}} \Omega^{\mu+\frac{1}{2}}} \int_{0}^{\infty}\left(\frac{1}{\alpha}\right)^{m_{s}+\mu+\frac{3}{2}} \\
& \times \exp \left(-\frac{2 \mu h r^{2}}{\alpha \Omega}-\frac{m_{s}}{\alpha}\right) I_{\mu-\frac{1}{2}}\left(\frac{2 \mu H r^{2}}{\alpha \Omega}\right) \mathrm{d} \alpha .
\end{aligned}
$$

Performing a simple transformation of variables and applying [46, Eq. (2.15.3.2)] along with some algebraic manipulation, (41) can be expressed in closed-form as given in (12).

\section{B. Proof of Theorem 6}

By expanding the Gauss hypergeometric function in terms of the series representation ${ }^{5}$ [31, Eq. (07.23.02.0001.01)], the CDF of the instantaneous SNR of the $\eta-\mu /$ inverse gamma model can be expressed as

$$
\begin{aligned}
& F_{\gamma}(\gamma)=\int_{0}^{\gamma} \frac{2^{2 \mu} \mu^{2 \mu} h^{\mu}\left(m_{s} \bar{\gamma}\right)^{m_{s}} \gamma^{2 \mu-1}}{B\left(m_{s}, 2 \mu\right)\left(2 \mu h \gamma+m_{s} \bar{\gamma}\right)^{m_{s}+2 \mu}} \\
& \quad \times \sum_{i=0}^{\infty} \frac{\left(\frac{m_{s}+2 \mu}{2}\right)_{i}\left(\frac{m_{s}+2 \mu+1}{2}\right)_{i}}{i !\left(\frac{2 \mu+1}{2}\right)_{i}}\left(\frac{2 \mu H \gamma}{2 \mu h \gamma+m_{s} \bar{\gamma}}\right)^{2 i} \mathrm{~d} \gamma
\end{aligned}
$$

Using [26, Eq. (3.194.1)] and [31, Eq. (07.23.02.0001.01)], we obtain the CDF of the instantaneous SNR of the $\eta$ - $\mu$ / inverse gamma model as given in (14).

\section{Proof of Theorem 7}

The higher order moments of the instantaneous SNR of the proposed $\eta-\mu /$ inverse gamma composite fading model can be expressed using an exact infinite series expansion of the Gauss hypergeometric function [31, Eq. (07.23.02.0001.01)] as follows

$$
\begin{aligned}
& \mathbb{E}\left[\gamma^{n}\right]=\int_{0}^{\infty} \frac{2^{2 \mu} \mu^{2 \mu} h^{\mu}\left(m_{s} \bar{\gamma}\right)^{m_{s}} \gamma^{2 \mu+n-1}}{B\left(m_{s}, 2 \mu\right)\left(2 \mu h \gamma+m_{s} \bar{\gamma}\right)^{m_{s}+2 \mu}} \\
& \quad \times \sum_{i=0}^{\infty} \frac{\left(\frac{m_{s}+2 \mu}{2}\right)_{i}\left(\frac{m_{s}+2 \mu+1}{2}\right)_{i}}{i !\left(\frac{2 \mu+1}{2}\right)_{i}}\left(\frac{2 \mu H \gamma}{2 \mu h \gamma+m_{s} \bar{\gamma}}\right)^{2 i} \mathrm{~d} \gamma
\end{aligned}
$$

${ }^{5}$ For $|z|<1$, the Gauss hypergeometric function, ${ }_{2} F_{1}(\cdot, \cdot ; \cdot ; z)$, is defined as a convergent infinite sum. In this case, as shown in (13), $z=\left(\frac{2 \mu H \gamma}{2 \mu h \gamma+m_{s} \bar{\gamma}}\right)^{2}$. Since $h=\left(2+\eta^{-1}+\eta\right) / 4, H=\left(\eta^{-1}-\eta\right) / 4$ and $\mu, \gamma, m_{s}, \bar{\gamma}>0$, the denominator $\left(2 \mu h \gamma+m_{s} \bar{\gamma}\right)$ is always greater than the numerator $(2 \mu H \gamma)$ and thus $z$ is always smaller than 1 .
With the aid of [26, Eq. (3.194.3)] and the series representation of the Gauss hypergeometric function [31, Eq. (07.23.02.0001.01)], the higher order moments of the instantaneous SNR of the $\eta-\mu$ / inverse gamma model can be obtained in closed-form as given in (19).

\section{Proof of Corollary 2}

Substituting $n=1$ and $n=2$ in (19), we obtain the first and second order moments as follows

$$
\mathbb{E}[\gamma]=\frac{B\left(m_{s}-1,2 \mu+1\right)}{h^{\mu} B\left(m_{s}, 2 \mu\right)}\left(\frac{m_{s} \bar{\gamma}}{2 \mu h}\right){ }_{2} F_{1}\left(\mu+\frac{1}{2}, \mu+1 ; \mu+\frac{1}{2} ; \frac{H^{2}}{h^{2}}\right)
$$

and

$\mathbb{E}\left[\gamma^{2}\right]=\frac{B\left(m_{s}-2,2 \mu+2\right)}{h^{\mu} B\left(m_{s}, 2 \mu\right)}\left(\frac{m_{s} \bar{\gamma}}{2 \mu h}\right)_{2}^{2} F_{1}\left(\mu+1, \mu+\frac{3}{2} ; \mu+\frac{1}{2} ; \frac{H^{2}}{h^{2}}\right)$.

Using ${ }_{2} F_{1}(c-a, c-b ; c ; z)=(1-z)^{a+b-c}{ }_{2} F_{1}(a, b ; c ; z)$ [31, Eq. (07.23.17.0054.01)], the Gauss hypergeometric functions in (44) and (45) can be rewritten as

$$
\begin{aligned}
{ }_{2} F_{1} & \left(\mu+\frac{1}{2}, \mu+1 \mu+\frac{1}{2} ; \frac{H^{2}}{h^{2}}\right) \\
& =\left(1-\frac{H^{2}}{h^{2}}\right)^{-(\mu+1)}{ }_{2} F_{1}\left(0,-\frac{1}{2} ; \mu+\frac{1}{2} ; \frac{H^{2}}{h^{2}}\right)
\end{aligned}
$$

and

$$
\begin{aligned}
{ }_{2} F_{1} & \left(\mu+1, \mu+\frac{3}{2} ; \mu+\frac{1}{2} ; \frac{H^{2}}{h^{2}}\right) \\
& =\left(1-\frac{H^{2}}{h^{2}}\right){ }_{2}^{-(\mu+2)} F_{1}\left(-\frac{1}{2},-1 ; \mu+\frac{1}{2} ; \frac{H^{2}}{h^{2}}\right) .
\end{aligned}
$$

By substituting the series expansion of the Gauss hypergeometric function [31, Eq. (07.23.02.0001.01)] and then simplifying the Pochhammer terms, we can obtain ${ }_{2} F_{1}\left(0,-\frac{1}{2} ; \mu+\frac{1}{2} ; \frac{H^{2}}{h^{2}}\right)=1$ and ${ }_{2} F_{1}\left(-\frac{1}{2},-1 ; \mu+\frac{1}{2} ; \frac{H^{2}}{h^{2}}\right)=$ $\left(h^{2}+H^{2}+2 \mu h^{2}\right) /\left(h^{2}(1+2 \mu)\right)$. Now substituting (46) and (47) into (44) and (45) respectively and carrying out some algebraic manipulation, we obtain the AF of the $\eta-\mu /$ inverse gamma model in closed-form as given in (20).

\section{E. Proof of Theorem 8}

The MGF can be derived from (13) using an infinite series expansion of the Gauss hypergeometric function [31, Eq. (07.23.02.0001.01)]

$$
\begin{aligned}
& M_{\gamma}(-s)=\int_{0}^{\infty} \frac{(2 \mu)^{2 \mu} h^{\mu}\left(m_{s} \bar{\gamma}\right)^{m_{s}} \gamma^{2 \mu-1} \exp (-s \gamma)}{B\left(m_{s}, 2 \mu\right)\left(2 \mu h \gamma+m_{s} \bar{\gamma}\right)^{m_{s}+2 \mu}} \\
& \quad \times \sum_{i=0}^{\infty} \frac{\left(\frac{m_{s}+2 \mu}{2}\right)_{i}\left(\frac{m_{s}+2 \mu+1}{2}\right)_{i}}{i !\left(\frac{2 \mu+1}{2}\right)_{i}}\left(\frac{2 \mu H \gamma}{2 \mu h \gamma+m_{s} \bar{\gamma}}\right)^{2 i} \mathrm{~d} \gamma
\end{aligned}
$$

Using [26, Eq. (3.383.5)] and [31, Eq. (07.03.02.0001.01)], we obtain the MGF of the $\eta-\mu$ / inverse gamma model as given in (21), which completes the proof.

\section{REFERENCES}

[1] M. K. Simon and M.-S. Alouini, Digital communication over fading channels, 2nd ed. New York: Wiley, 2005. 
[2] A. Abdi, W. C. Lau, M.-S. Alouini, and M. Kaveh, "A new simple model for land mobile satellite channels: First-and second-order statistics," IEEE Trans. Wireless Commun., vol. 2, no. 3, pp. 519-528, May 2003.

[3] C. Loo, "A statistical model for a land mobile satellite link," IEEE Trans. Veh. Technol., vol. 34, no. 3, pp. 122-127, Aug. 1985.

[4] G. E. Corazza and F. Vatalaro, "A statistical model for land mobile satellite channels and its application to nongeostationary orbit systems," IEEE Trans. Veh. Technol., vol. 43, no. 3, pp. 738-742, Aug. 1994.

[5] F. Hansen and F. I. Meno, "Mobile fading - Rayleigh and lognormal superimposed," IEEE Trans. Veh. Technol., vol. 26, no. 4, pp. 332-335, Nov. 1977.

[6] H. Suzuki, "A statistical model for urban radio propogation," IEEE Trans. Commun., vol. 25, no. 7, pp. 673-680, Jul. 1977.

[7] T. T. Tjhung and C. C. Chai, "Fade statistics in microcellular mobile radio channels with shadowing," in Proc. IEEE ICC, Jun. 1998, pp. $1645-1649$.

[8] A. Abdi and M. Kaveh, "On the utility of gamma PDF in modeling shadow fading (slow fading)," in Proc. IEEE VTC, vol. 3, May 1999, pp. $2308-2312$.

[9] I. M. Kostić, "Analytical approach to performance analysis for channel subject to shadowing and fading," IEE Proc. Comm., vol. 152, no. 6, pp. 821-827, Dec. 2005.

[10] A. Abdi and M. Kaveh, " $K$ distribution: An appropriate substitute for Rayleigh-lognormal distribution in fading-shadowing wireless channels," IET Electron. Lett., vol. 34, no. 9, pp. 851-852, Apr. 1998.

[11] A. H. Marcus, "Power sum distributions: An easier approach using the Wald distribution," J. Am. Stat. Assoc., vol. 71, no. 353, pp. 237-238, Mar. 1976.

[12] Karmeshu and R. Agrawal, "On efficacy of Rayleigh-inverse Gaussian distribution over $K$-distribution for wireless fading channels," Wireless Commun. Mobile Comput., vol. 7, no. 1, pp. 1-7, 2007.

[13] T. Eltoft, "The Rician inverse Gaussian distribution: A new model for non-Rayleigh signal amplitude statistics," IEEE Trans. Image Process., vol. 14, no. 11, pp. 1722-1735, Nov. 2005.

[14] A. Laourine, M.-S. Alouini, S. Affes, and A. Stéphenne, "On the performance analysis of composite multipath/shadowing channels using the G-distribution," IEEE Trans. Commun., vol. 57, no. 4, pp. $1162-$ 1170, Apr. 2009.

[15] P. C. Sofotasios, T. A. Tsiftsis, K. H. Van, S. Freear, L. R. Wilhelmsson, and M. Valkama, "The $\kappa-\mu$ / IG composite statistical distribution in RF and FSO wireless channels," in Proc. IEEE VTC, Sep. 2011, pp. 1-5.

[16] P. C. Sofotasios, T. A. Tsiftsis, M. Ghogho, L. R. Wilhelmsson, and M. Valkama, "The $\eta-\mu$ / IG distribution: A novel physical multipath/shadowing fading model," in Proc. IEEE ICC, Jun. 2013, pp. 57155719.

[17] S. L. Cotton, "A statistical model for shadowed body-centric communications channels: theory and validation," IEEE Trans. Antennas Propag., vol. 62, no. 3, pp. 1416-1424, Mar. 2014.

[18] J. F. Paris, "Statistical characterization of $\kappa-\mu$ shadowed fading," IEEE Trans. Veh. Technol., vol. 63, no. 2, pp. 518-526, Feb. 2014.

[19] P. C. Sofotasios and S. Freear, "On the $\kappa-\mu /$ gamma composite distribution: A generalized multipath/shadowing fading model," in Proc. IEEE IMOC, Nov. 2011, pp. 390-394.

[20] S. K. Yoo, S. L. Cotton, P. C. Sofotasios, and S. Freear, "Shadowed fading in indoor off-body communication channels: A statistical characterization using the $\kappa-\mu$ / gamma composite fading model," IEEE Trans. Wireless Commun., vol. 15, no. 8, pp. 5231-5244, Aug. 2016.

[21] P. C. Sofotasios and S. Freear, "The $\eta-\mu /$ gamma composite fading model," in Proc. IEEE ICWITS, Sep. 2010, pp. 1-4

[22] J. Zhang, M. Matthaiou, Z. Tan, and H. Wang, "Performance analysis of digital communication systems over composite $\eta-\mu /$ gamma fading channels," IEEE Trans. Veh. Technol., vol. 61, no. 7, pp. 3114-3124, Sep. 2012

[23] S. K. Yoo, S. L. Cotton, P. C. Sofotasios, M. Matthaiou, M. Valkama, and G. K. Karagiannidis, "The $\kappa-\mu$ / inverse gamma fading model," in Proc. IEEE PIMRC, Sep. 2015, pp. 425-429.

[24] S. K. Yoo, P. C. Sofotasios, S. L. Cotton, M. Matthaiou, M. Valkama, and G. K. Karagiannidis, "The $\eta-\mu$ / inverse gamma composite fading model," in Proc. IEEE PIMRC, Sep. 2015, pp. 166-170.

[25] M. D. Yacoub, "The $\kappa-\mu$ distribution and the $\eta-\mu$ distribution," IEEE Antennas Propag. Mag., vol. 49, no. 1, pp. 68-81, Feb. 2007.

[26] I. S. Gradshteyn and I. M. Ryzhik, Table of Integrals, Series, and Products, 7th ed. London: Academic Press, 2007.

[27] G. L. Stüber, Principles of mobile communication, 3rd ed. Springer, 2011.

[28] C. Forbes, M. Evans, N. Hastings, and B. Peacock, Statistical distributions. John Wiley \& Sons, 2011.
[29] Wolfram Research, Inc., 2017, visited on 03/26/2017. [Online]. Available: http://functions.wolfram.com/Notations/5/.

[30] P. Humbert, "Sur les fonctions hypercylindriques," C. R. Acad. Sci., Paris, vol. 171, pp. 490-492, 1920.

[31] Wolfram Research, Inc., 2017, visited on 03/26/2017. [Online]. Available: http://functions.wolfram.com/id.

[32] J. W. Jhuang and H. P. Ma, "A patch-sized wearable ECG/respiration recording platform with DSP capability," in Proc. HealthCom, 2015, pp. 298-304.

[33] Y. Koyama and K. Watanabe, "Auditory golf coaching system using perceptive sportswear based on hetero-core optics," in Proc. IEEE GCCE, Oct. 2014, pp. 643-646.

[34] S. L. Cotton, W. G. Scanlon, and B. K. Madahar, "Millimeter-wave soldier-to-soldier communications for covert battlefield operations," IEEE Commun. Mag., vol. 47, no. 10, pp. 72-81, Oct. 2009.

[35] D. Arsenault and A. Whitehead, "Wearable sensor networks for motion capture," in Proc. IEEE INTETAIN, Jun. 2015, pp. 158-167.

[36] T. S. Rappaport, Wireless communications: Principles and practice. New Jersey: Prentice Hall PTR, 2002.

[37] D. H. Johnson and S. Sinanovic, Symmetrizing the Kullback-Leibler distance. Rice University Working Paper, 2001.

[38] S. Kullback, Information theory and statistics. Courier Corporation, 1997.

[39] S. K. Yoo, S. L. Cotton, W. G. Scanlon, and G. A. Conway, "An experimental evaluation of switched combining based macro-diversity for wearable communications operating in an outdoor environment," IEEE Trans. Wireless Commun., vol. 16, no. 8, pp. 5338-5352, Aug. 2017.

[40] K. Doppler, M. Rinne, C. Wijting, C. B. Ribeiro, and K. Hugl, "Deviceto-device communication as an underlay to LTE-advanced networks," IEEE Commun. Mag., vol. 47, no. 12, pp. 42-49, Dec. 2009.

[41] B. Kaufman, J. Lilleberg, and B. Aazhang, "Spectrum sharing scheme between cellular users and ad-hoc device-to-device users," IEEE Trans. Wireless Commun., vol. 12, no. 3, pp. 1038-1049, Mar. 2013.

[42] G. George, R. K. Mungara, and A. Lozano, "An analytical framework for device-to-device communication in cellular networks," IEEE Trans. Wireless Commun., vol. 14, no. 11, pp. 6297-6310, Jul. 2015.

[43] J. Karedal et al., "A geometry-based stochastic MIMO model for vehicle-to-vehicle communications," IEEE Trans. Wireless Commun. vol. 8, no. 7, pp. 3646-3657, Jul. 2009.

[44] L. Shi and K. W. Sung, "Spectrum requirement for vehicle-to-vehicle communication for traffic safety," in Proc. IEEE VTC, May 2014, pp. $1-5$.

[45] M. Abramowitz and I. A. Stegun, Handbook of mathematical functions. Washington, DC, US Dept. of Commerce, National Bureau of Standards, 1972.

[46] A. P. Prudnikov, Y. A. Brychkov, and O. I. Marichev, Integrals and Series, Volume 2: Special Functions. Moscow: Fiziko-Matematicheskaya Literatura, 2003.

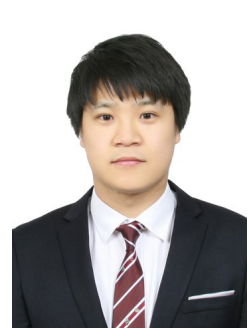

Seong Ki Yoo (S'14-M'17) received the B.Eng. (Hons.) degree in telecommunication systems from the University of Surrey, Guildford, U.K., in 2010, and the M.Sc. degree in communications and signal processing from Imperial College London, London, U.K., in 2012 and the Ph.D. degree from Queen's University Belfast, Belfast, U.K., in 2017. His Doctoral studies were sponsored by U.K. EPSRC. He was a junior researcher with Korea Electrotechnology Research Institute (KERI), Ansan, South Korea, from 2012 to 2013. He is currently a Post-Doctoral Research Fellow with Centre for Wireless Innovation, ECIT Institute, Queen's University Belfast. His research interests are in the areas of fading channel characterization and modeling for wearable communications and diversity in wearable applications.

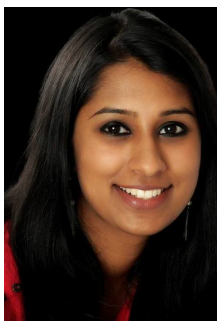

Nidhi Bhargav (S'15) graduated from Visvesvaraya Technological University, Karnataka, India, with a B.E in Telecommunications Engineering (with distinction), in 2011. She then received the M.Sc. degree (with distinction) in wireless communications and signal processing from the University of Bristol, UK, in 2012. She is currently a Ph.D. student at Queen's University of Belfast, UK. Her research interests include physical layer security, channel characterization and modeling, cellular device-todevice and body-centric communications. 


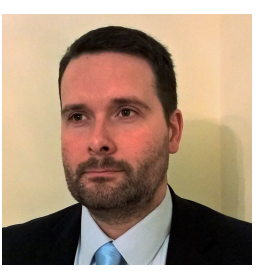

Simon L. Cotton (S'04-M'07-SM'14) received the B.Eng. degree in electronics and software from the University of Ulster, Ulster, U.K., in 2004 and the $\mathrm{Ph} . \mathrm{D}$. degree in electrical and electronic engineering from Queen's University of Belfast, Belfast, U.K., in 2007. He is currently a Reader in wireless communications with the Institute of Electronics, Communications and Information Technology (ECIT), Queen's University Belfast. He is also a Co-founder of and the Chief Technology Officer with ActivWireless Ltd, Belfast, U.K. He has authored and co-authored over 100 publications in major IEEE/IET journals and refereed international conferences, two book chapters, and two patents. Among his research interests are cellular device-to-device, vehicular, and wearable communications. His other research interests include radio channel characterization and modeling and the simulation of wireless channels. Dr. Cotton was awarded the H. A. Wheeler Prize, in July 2010, by the IEEE Antennas and Propagation Society for the best applications journal paper in the IEEE TRANSACTIONS ON ANTENNAS AND PROPAGATION during 2009. In July 2011, he was awarded the Sir George Macfarlane Award from the U.K Royal Academy of Engineering in recognition of his technical and scientific attainment since graduating from his first degree in engineering.

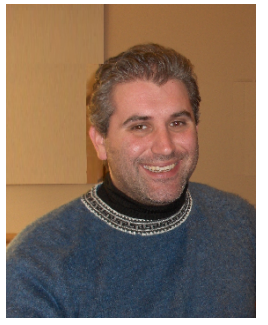

Paschalis C. Sofotasios (S'07-M'12-SM'16) was born in Volos, Greece in 1978. He received the MEng degree from Newcastle University, UK, in 2004, the MSc degree from the University of Surrey, UK, in 2006, and the $\mathrm{PhD}$ degree from the University of Leeds, UK, in 2011. His MSc studies were funded by a scholarship from UK-EPSRC and his Doctoral studies were sponsored by UK-EPSRC and Pace plc. He has held academic positions at the University of Leeds, UK, the University of California, Los Angleles, USA, Tampere University of Technology, Finland, Khalifa University, UAE and the Aristotle University of Thessaloniki, Greece. His research interests are in the broad areas of digital and optical wireless communications including topics on pure mathematics. Dr. Sofotasios serves as a regular reviewer for several international journals and has been a Member of the Technical Program Committee of numerous IEEE conferences. He currently serves as an Editor of the IEEE COMMUNICATIONS LETTERS and he has received an Exemplary Reviewer award by IEEE COMMUNICATIONS LETTERS in 2012 and by IEEE TRANSACTIONS on COMMUNiCATIONS in 2015 and 2016. He is a Senior Member IEEE and a co-recipient of the best paper award at ICUFN' 13.

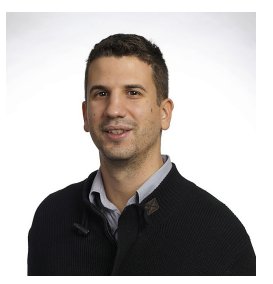

Michail Matthaiou (S'05-M'08-SM'13) was born in Thessaloniki, Greece in 1981. He obtained the Diploma degree (5 years) in Electrical and Computer Engineering from the Aristotle University of Thessaloniki, Greece in 2004. He then received the M.Sc. (with distinction) in Communication Systems and Signal Processing from the University of Bristol, U.K. and Ph.D. degrees from the University of Edinburgh, U.K. in 2005 and 2008, respectively. From September 2008 through May 2010, he was with the Institute for Circuit Theory and Signal Processing, Munich University of Technology (TUM), Germany working as a Postdoctoral Research Associate. He is currently a Reader (equivalent to Associate Professor) in Multiple-Antenna Systems at Queen's University Belfast, U.K. after holding an Assistant Professor position at Chalmers University of Technology, Sweden. His research interests span signal processing for wireless communications, massive MIMO, hardware-constrained communications, and performance analysis of fading channels.

Dr. Matthaiou will receive the 2017 IEEE Communications Society Leonard G. Abraham Prize. He was the recipient of the 2011 IEEE ComSoc Best Young Researcher Award for the Europe, Middle East and Africa Region and a co-recipient of the 2006 IEEE Communications Chapter Project Prize for the best M.Sc. dissertation in the area of communications. He was corecipient of the Best Paper Award at the 2014 IEEE International Conference on Communications (ICC) and was an Exemplary Reviewer for IEEE COMMUNiCATIONS LETTERS for 2010. In 2014, he received the Research Fund for International Young Scientists from the National Natural Science Foundation of China. In the past, he was an Associate Editor for the IEEE TRANSACTIONS ON COMMUNICATIONS, Associate Editor/Senior Editor for IEEE COMMUNICATIONS LETTERS and was the Lead Guest Editor of the special issue on "Large-scale multiple antenna wireless systems" of the IEEE Journal on SElected AREas in Communications. He was the chair of the Wireless Communications Symposium (WCS) at IEEE GLOBECOM 2016.

0090-6778 (c) 2017 IEEE. Translations and content mining are permitted for academic research only. Personal use is also permitted, but republication/redistribution requires IEEE permission. See http://www.ieee.org/publications_standards/publications/rights/index.html for more information.

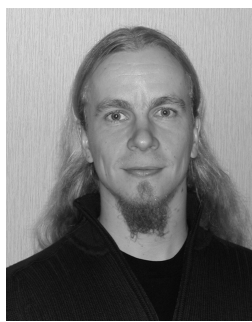

Mikko Valkama (S00M01SM15) was born in Pirkkala, Finland, on November 27, 1975. He received the M.Sc. and Ph.D. Degrees (both with honors) in electrical engineering (EE) from Tampere University of Technology (TUT), Finland, in 2000 and 2001, respectively. In 2002, he received the Best Ph.D. Thesis-award by the Finnish Academy of Science and Letters for his dissertation entitled "Advanced I/Q signal processing for wideband receivers: Models and algorithm". In 2003, he was working as a visiting post-doc research fellow with the Communications Systems and Signal Processing Institute at SDSU, San Diego, CA. Currently, he is a Full Professor and Laboratory Head at the Laboratory of Electronics and Communications Engineering at TUT, Finland. His general research interests include radio communications, communications signal processing, estimation and detection techniques, signal processing algorithms for flexible radios, cognitive radio, full-duplex radio, radio localization, and $5 \mathrm{G}$ and beyond mobile radio networks.

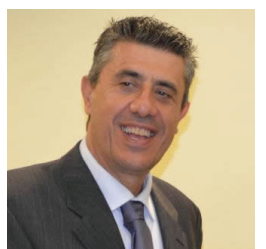

George K. Karagiannidis (M'96-SM'03-F'14) was born in Pithagorion, Samos Island, Greece. He received the University Diploma (5 years) and $\mathrm{PhD}$ degree, both in electrical and computer engineering from the University of Patras, in 1987 and 1999, respectively. From 2000 to 2004, he was a Senior Researcher at the Institute for Space Applications and Remote Sensing, National Observatory of Athens, Greece. In June 2004, he joined the faculty of Aristotle University of Thessaloniki, Greece where he is currently Professor in the Electrical \& Computer Engineering Dept. and Director of Digital Telecommunications Systems and Networks Laboratory. He is also Honorary Professor at South West Jiaotong University, Chengdu, China. His research interests are in the broad area of Digital Communications Systems and Signal processing, with emphasis on Wireless Communications, Optical Wireless Communications, Wireless Power Transfer and Applications, Molecular and Nanoscale Communications, Stochastic Processes in Biology and Wireless Security. He is the author or co-author of more than 450 technical papers published in scientific journals and presented at international conferences. He is also author of the Greek edition of a book on "Telecommunications System" and co-author of the book "Advanced Optical Wireless Communications Systems", Cambridge Publications, 2012. Dr. Karagiannidis has been involved as General Chair, Technical Program Chair and member of Technical Program Committees in several IEEE Communications, Senior Editor of IEEE Communications Letters, Editor of the EURASIP Journal of Wireless Communications \& Networks and several times Guest Editor in IEEE Selected Areas in Communications. From 2012 to 2015 he was the Editor-in Chief of IEEE Communications Letters. Dr. Karagiannidis is IEEE Fellow and one of the highly-cited authors across all areas of Electrical Engineering, recognized as 2015, 2016 and 2017 Web-ofScience Highly-Cited Researcher. and non-IEEE conferences. In the past, he was Editor in IEEE Transactions on 